\title{
QUASIPLURISUBHARMONIC GREEN FUNCTIONS
}

\author{
DAN COMAN AND VINCENT GUEDJ
}

\begin{abstract}
Given a compact Kähler manifold $X$, a quasiplurisubharmonic function is called a Green function with pole at $p \in X$ if its Monge-Ampère measure is supported at $p$. We study in this paper the existence and properties of such functions, in connection to their singularity at $p$. A full characterization is obtained in concrete cases, such as (multi)projective spaces.
\end{abstract}

\section{INTRODUCTION}

Let $X$ be a compact Kähler manifold of complex dimension $n$. We pursue the study started in $[\mathrm{Y}],[\mathrm{Ko} 1]$, [Ko2], [GZ2], [EGZ], [BGZ] of the range of the complex Monge-Ampère operator. Given a Kähler class $\alpha \in H^{1,1}(X, \mathbb{R})$ and a positive Radon measure $\mu$, the problem is to solve the equation $T^{n}=\mu$, where $T$ is a positive closed $(1,1)$-current in $\alpha$. When $\mu$ does not charge pluripolar sets, a complete answer was given in [GZ2]. The main purpose of this article is to start and study the case when $\mu$ charges pluripolar sets by looking at measures $\mu$ which are sums of Dirac masses. The equation now reads

$$
T^{n}=\sum_{j=1}^{k} c_{j} \delta_{p_{j}} .
$$

We seek solution(s) $T \in \alpha$ whose potentials are locally bounded away from the poles $p_{1}, \ldots, p_{k}$. An obvious necessary condition in order to solve (1) is that the volume of $\alpha$,

$$
V_{\alpha}:=\operatorname{Vol}(\alpha)=\alpha^{n},
$$

is equal to the total mass of $\mu, \mu(X)=\sum c_{j}=\operatorname{Vol}(\alpha)$.

Fix $\theta$ a Kähler form representing $\alpha$ and let $P S H(X, \theta)$ denote the set of $\theta$ plurisubharmonic $\left(\theta\right.$-psh) functions: these are functions $\varphi \in L^{1}(X, \mathbb{R})$ which are upper semicontinuous and such that $T=\theta+d d^{c} \varphi$ is a positive current. Here $d=\partial+\bar{\partial}$ and $d^{c}=\frac{1}{2 \pi i}(\partial-\bar{\partial})$. Solving (1) is therefore equivalent to finding a "quasiplurisubharmonic Green function":

2000 Mathematics Subject Classification. Primary: 32U35. Secondary: 32W20, 32Q15.

Key words and phrases. (quasi)plurisubharmonic functions; positive closed currents; Lelong numbers; Kähler manifolds.

D. Coman was supported by the NSF grant DMS 0500563. Support by the Institut Mittag-Leffler (Djursholm, Sweden) is gratefully acknowledged. 
Definition. A function $\varphi \in P S H(X, \theta)$ is called a $\theta$-psh Green function with (isolated) poles at $p_{1}, \ldots, p_{k} \in X$ if it is locally bounded in $X \backslash\left\{p_{1}, \ldots, p_{k}\right\}$ and

$$
\left(\theta+d d^{c} \varphi\right)^{n}=V_{\alpha} \sum_{j=1}^{k} m_{j} \delta_{p_{j}}, \text { where } m_{j}>0, \sum_{j=1}^{k} m_{j}=1 .
$$

In [CGZ], the domain $D M A(X, \theta)$ of the Monge-Ampère operator was defined as the largest set of $\theta$-psh functions on which the operator is continuous along decreasing sequences of bounded $\theta$-psh functions. Hence one can consider a more general notion of $\theta$-psh Green function, by only requiring in the above definition that $\varphi \in D M A(X, \theta)$, instead of $\varphi$ being locally bounded away from the poles. We will not pursue this here.

Similar objects were considered by several authors in a local context ([Lm], [Kl], [D1], [Le], [CP], [Co1], [CN]), and have found important applications (see e.g. [BP], $[\mathrm{He}],[\mathrm{DH}])$. In our global context their existence depends on the geometry of $X$ and on the local positivity properties of $\alpha$ at the poles.

We therefore study in section 1 several indicators of the local positivity properties of $\alpha$, following Demailly [D2]. Recall that the Lelong number $\nu(\varphi, x)$ of a $\theta$-psh function $\varphi$ at $x$ is the largest constant $\nu$ for which $\varphi(p) \leq \nu \log \operatorname{dist}(p, x)+O(1)$ holds for $p$ near $x$. If $\varphi(p)=\nu \log \operatorname{dist}(p, x)+O(1)$ for $p$ near $x$ and $\nu>0$, we say that $\varphi$ has an isotropic pole at $x$ with Lelong number $\nu$.

We let $\nu(\alpha, x)$ (resp. $\varepsilon(\alpha, x)$ ) denote the maximal (resp. maximal isotropic) logarithmic singularity that a positive closed current $T \in \alpha$ can have at the point $x$. The indicator $\varepsilon(\alpha, x)$, introduced by Demailly [D2], is called the Seshadri constant of $\alpha$ at $x$ and was intensively studied in algebraic geometry. We note in section 1 that for all $x \in X$,

$$
\nu(\alpha, x) \geq \operatorname{Vol}(\alpha)^{1 / n} \geq \varepsilon(\alpha, x) .
$$

Thus a necessary condition for the existence of a $\alpha$-Green function with one isotropic pole at $x$ is that $\operatorname{Vol}(\alpha)^{1 / n}=\varepsilon(\alpha, x)$. This is far from being true in general: we observe for instance in Proposition 3.1 that this is never the case when $X$ is a multiprojective space. Even if this condition is satisfied, it is not clear whether it is sufficient, nor is it clear that the supremum in the definition of $\varepsilon$ is attained. We observe in section 4.3.2 that the following properties are equivalent:

- existence of a Green function with 9 isotropic poles in general position in $\mathbb{P}^{2}$

- existence of a Green function with one isotropic pole in generic position on a degree 1 Del Pezzo surface;

- existence of a positive metric with bounded potentials for $c_{1}(Y)$, where $Y \rightarrow$ $\mathbb{P}^{2}$ denotes the blow up of $\mathbb{P}^{2}$ at 9 points in general position,

the last one being a famous open problem [DPS]. We therefore introduce in section 1 weaker notions of Green functions. We show in Theorems 1.4, 1.5 and Proposition 1.6 how to construct these by a balayage procedure. It is a delicate and interesting problem to determine whether $\theta$-psh Green functions always exist. As already observed, we have to consider arbitrary singularities. The balayage procedure depends on the choice of local data $\left(u_{1}, \ldots, u_{k}\right)$ encoding the singularities at the poles $\left(p_{1}, \ldots, p_{k}\right)$. In particular, the problem of constructing $\theta$-psh Green functions is 
reduced to finding local data for which the functions $g$ constructed in Theorems 1.4 and 1.5 have isolated singularities at $p_{j}$.

In section 2 we give a complete description of all these notions on the complex projective space $\mathbb{P}^{n}$. In particular, we characterize in Theorem 2.4 Green functions arising naturally from rational maps $f: \mathbb{P}^{n} \rightarrow \mathbb{P}^{n-1}$ with finite indeterminacy set. We end section 2 by constructing interesting dynamical Green functions.

In section 3 we compute similar quantities for multiprojective spaces, focusing on $\mathbb{P}^{1} \times \mathbb{P}^{1}$. We show in Proposition 3.4 that Green functions with one pole correspond to a certain class of Green functions with three poles on $\mathbb{P}^{2}$. A large class of examples of these can be constructed using Theorem 2.4 (see Example 3.5). However, there is no Green function with one isotropic pole on $\mathbb{P}^{1} \times \mathbb{P}^{1}$ (Corollary 3.2).

In section 4 we turn our attention to the case of smooth Del Pezzo surfaces, focusing on those of degree 1, i.e. blow ups $X$ of $\mathbb{P}^{2}$ at 8 points in general position. Let $\alpha$ be the first Chern class of $X$. We prove in Proposition 4.1 that $\nu(\alpha, x)=1$ if $x \in X \backslash S$, and $\nu(\alpha, x)=2$ if $x \in S$. Here $S$ is the set of singular points on the singular cubics passing through the 8 blown up points, and $1 \leq|S| \leq 12$. The results of Proposition 4.1 allow us to compute, using currents, the exact value of Tian's " $\alpha$ invariant", and to deduce that $X$ has a Kähler-Einstein metric (section 4.2). We conclude the paper with the discussion in section 4.3 of $\omega$-psh Green functions with one pole $x \in X$, where $\omega \in \alpha$ is a Kähler form. Such functions are easy to construct when $x \in S$. For generic points $x \notin S$ the existence of Green functions with an isotropic pole at $x$ of maximal Lelong number $1=\varepsilon(\alpha, x)$ is equivalent to a famous open problem in algebraic geometry (see section 4.3.2).

Acknowledgement. We would like to thank the referee for his comments which helped improve the exposition of this paper.

\section{Local positivity of $(1,1)$ Classes and Green functions}

Let $\mathcal{P}(X)$ be the set of all positive closed currents of bidegree $(1,1)$ on $X$. For $\alpha \in H^{1,1}(X, \mathbb{R})$ we let

$$
\mathcal{P}(\alpha)=\{T \in \mathcal{P}(X): T \in \alpha\}
$$

be the set of positive closed currents whose cohomology class is $\alpha$. By definition, a class $\alpha$ is pseudoeffective if $\mathcal{P}(\alpha) \neq \emptyset$. Let $H_{p s e f}^{1,1}(X, \mathbb{R})$ denote the closed convex cone of all pseudoeffective $(1,1)$ classes.

There are two other interesting cones in $H_{p s e f}^{1,1}(X, \mathbb{R})$ which correspond to stronger notions of positivity. We let $H_{\text {Kaehler }}^{1,1}(X, \mathbb{R})$ denote the cone of Kähler classes and $H_{\text {nef }}^{1,1}(X, \mathbb{R})$ denote its closure. Then $H_{\text {Kaehler }}^{1,1}(X, \mathbb{R})$ is the interior of $H_{n e f}^{1,1}(X, \mathbb{R})$.

Following Demailly [D2], we would like to measure the local positivity of a class $\alpha$. There are two main indicators, in connection to the various types of positivity. In the sequel we denote by $\nu(T, x)$ the Lelong number of $T \in \mathcal{P}(X)$ at a point $x$.

Definition 1.1. Let $\pi: \widetilde{X} \rightarrow X$ denote the blow up of $X$ at a point $x$, and let $E=\pi^{-1}(x)$ denote the exceptional divisor.

1) For $\alpha \in H_{p s e f}^{1,1}(X, \mathbb{R})$ we set

$$
\nu(\alpha, x):=\sup \left\{\nu \geq 0: \pi^{\star} \alpha-\nu E \in H_{p s e f}^{1,1}(\widetilde{X}, \mathbb{R})\right\} .
$$


2) For $\alpha \in H_{n e f}^{1,1}(X, \mathbb{R})$ we set

$$
\varepsilon(\alpha, x):=\sup \left\{\varepsilon \geq 0: \pi^{\star} \alpha-\varepsilon E \in H_{n e f}^{1,1}(\tilde{X}, \mathbb{R})\right\} .
$$

The indicator $\nu(\alpha, x)$ is the maximal Lelong number that a current $T \in \mathcal{P}(\alpha)$ can have at $x$. In this case the supremum is attained, because $\mathcal{P}(\alpha)$ is a compact set (in the weak topology of currents).

The indicator $\varepsilon(\alpha, x)$ is called the Seshadri constant of $\alpha$ at $x$. It has been intensively studied since it was introduced by Demailly. We refer the reader to [La, Chapter 5] for a detailed account of this notion.

By definition we have $0 \leq \varepsilon(\alpha, x) \leq \nu(\alpha, x)$. It follows from the characterization of the Kähler cone obtained in $[\mathrm{DP}]$ that if $\alpha \in H_{n e f}^{1,1}(X, \mathbb{R})$ and $x \in X$ then

$$
\varepsilon(\alpha, x)=\min _{V}\left(\frac{\left(\alpha^{\operatorname{dim} V} \cdot V\right)}{\operatorname{mult}_{x} V}\right)^{\frac{1}{\operatorname{dim} V}},
$$

where the minimum is taken over all irreducible subvarieties $V \subseteq X$ with $\operatorname{dim} V \geq 1$ and $x \in V$ (see e.g. Proposition 5.1.9 and Remark 1.5.32 in [La]). With $V=X$, this yields the estimate (recall that $V_{\alpha}=\operatorname{Vol}(\alpha)$ ):

$$
\varepsilon(\alpha, x) \leq V_{\alpha}^{1 / n}, \forall x \in X .
$$

On the other hand, it follows easily from Theorem 1.4 below that if $\alpha \in H_{\text {Kaehler }}^{1,1}(X, \mathbb{R})$

$$
\nu(\alpha, x) \geq V_{\alpha}^{1 / n}, \forall x \in X .
$$

Both bounds are sharp in the case of $\mathbb{P}^{n}$.

Remark 1.2. If $\alpha \in H^{2}(X, \mathbb{Z})$ is an integral class, then $\nu(\alpha, x) \geq V_{\alpha}^{1 / n} \geq 1$ for all $x \in X$. Note also that if $\alpha$ is very ample then $\varepsilon(\alpha, x) \geq 1$.

An alternate description of the Seshadri constant $\varepsilon(\alpha, x)$ can be given in terms the maximal Lelong number of currents in $\mathcal{P}(\alpha)$ whose potentials have an isolated singularity at $x[\mathrm{D} 2]$. Let $\alpha \in H_{\text {Kaehler }}^{1,1}(X, \mathbb{R})$ and $\theta$ be a Kähler form representing $\alpha$. It follows as in [D2, Theorem 6.4] that for every $x \in X$,

$$
\begin{aligned}
\varepsilon(\alpha, x) & =\sup \left\{\gamma: \exists \varphi \in P S H(X, \theta),\|\varphi-\gamma \log \operatorname{dist}(\cdot, x)\|_{L^{\infty}(X)}<+\infty\right\} \\
& =\sup \left\{\gamma: \exists \varphi \in P S H(X, \theta), \nu(\varphi, x)=\gamma, \varphi \in L_{l o c}^{\infty}(U \backslash\{x\})\right\},
\end{aligned}
$$

where $U$ is a neighborhood of $x$ depending on $\varphi$. Recall that $\operatorname{PSH}(X, \theta)$ is the set of $\theta$-psh functions. The set of normalized $\theta$-psh functions, for example by the condition $\max _{X} \varphi=0$, is isomorphic to $\mathcal{P}(\alpha)$ via $\varphi \rightarrow \theta+d d^{c} \varphi \in \mathcal{P}(\alpha)$. The fact that the two supremums are equal is straightforward. Moreover, in this case we have $\varepsilon(\alpha, x)>0$ for all $x \in X$.

We now list a few elementary properties of these numerical indicators.

Proposition 1.3. 1) The functions $\alpha \rightarrow \nu(\alpha, x), \varepsilon(\alpha, x)$ are homogeneous and superadditive (i.e. $\nu(\alpha+\beta, x) \geq \nu(\alpha, x)+\nu(\beta, x)$ ).

2) The function $x \rightarrow \nu(\alpha, x)$ is upper semicontinuous.

3) If $\alpha$ is Kähler the function $x \rightarrow \varepsilon(\alpha, x)$ is lower semicontinuous. 
Proof. The upper semicontinuity property of $x \rightarrow \nu(\alpha, x)$ follows since $\mathcal{P}(\alpha)$ is compact and from the well known fact that $\lim \sup \nu\left(T_{j}, x_{j}\right) \leq \nu(T, x)$ as positive closed (1,1)-currents $T_{j} \rightarrow T$ and $x_{j} \rightarrow x$.

To prove 3), let $\theta \in \alpha$ be a Kähler form, $x \in X, 0<\epsilon<1$, and $0<\nu<\varepsilon(\alpha, x)$. We construct for all $y$ near $x$ a $\theta$-psh function $\varphi_{y}$ with $\varphi_{y}=(1-\epsilon) \nu \log \operatorname{dist}(\cdot, y)+$ $O(1)$. Using (3), this shows that $\liminf _{y \rightarrow x} \varepsilon(\alpha, y) \geq \varepsilon(\alpha, x)$.

By (3) there exists $\varphi \in P S H(X, \theta)$ such that $\varphi=\nu \log \operatorname{dist}(\cdot, x)+O(1)$. Let $B_{2} \subset \mathbb{C}^{n}$ be the ball of radius 2 centered at 0 . We can find a coordinate chart $f: B_{2} \longrightarrow U \subset X, f(0)=x$, and a function $\rho \in C^{\infty}(U)$ so that $d d^{c} \rho=\theta$ and

$$
\nu \log \|z\|-C \leq v(z):=(\rho+\varphi) \circ f(z) \leq \nu \log \|z\|+C, z \in B_{2},
$$

for some constant $C>0$. Fix $r>0$ small enough so that

$$
(1-\epsilon)\left(\nu \log \frac{r}{2}-2 C\right) \geq \nu \log r+2 C .
$$

Next, let $T_{w}$ be an automorphism of the unit ball $B_{1} \subset \mathbb{C}^{n}$ with $T_{w}(w)=0$. There exists $\delta(r)<r$ such that $\left\|T_{w}(z)\right\| \geq r / 2$, if $\|z\|=r$ and $\|w\|<\delta(r)$. For such $w$ we define the function $v_{w}$ on $B_{2}$ by

$$
v_{w}(z)=\left\{\begin{array}{l}
v(z)+C, 1 \leq\|z\|<2 \\
\max \left\{v(z)+C,(1-\epsilon)\left(v \circ T_{w}(z)-C\right)\right\}, r<\|z\|<1, \\
(1-\epsilon)\left(v \circ T_{w}(z)-C\right),\|z\| \leq r
\end{array}\right.
$$

Note that if $\|z\|=1$ then $v(z)+C \geq 0 \geq(1-\epsilon)\left(v \circ T_{w}(z)-C\right)$, while if $\|z\|=r$,

$$
(1-\epsilon)\left(v \circ T_{w}(z)-C\right) \geq(1-\epsilon)\left(\nu \log \frac{r}{2}-2 C\right) \geq \nu \log r+2 C \geq v(z)+C .
$$

Hence $v_{w}$ is psh on $B_{2}$ and $v(z)=(1-\epsilon) \nu \log \|z-w\|+O(1)$ for $z$ near $w$.

For $y=f(w)$, where $\|w\|<\delta(r)$, we finally let

$$
\varphi_{y}=\left\{\begin{array}{l}
\varphi+C, \text { on } X \backslash f\left(B_{1}\right), \\
v_{w} \circ f^{-1}-\rho, \text { on } f\left(B_{1}\right) .
\end{array}\right.
$$

Then $\varphi_{y}$ is $\theta$-psh and $\varphi_{y}=(1-\epsilon) \nu \log \operatorname{dist}(\cdot, y)+O(1)$ near $y$.

In general, the functions $\nu(\alpha, \cdot), \varepsilon(\alpha, \cdot)$ are not continuous (see e.g. Proposition 4.1 and section 4.3). Note that in the special case when $X$ is projective and $\alpha$ is an integral class, it follows from [La, Example 5.1.11] that $\varepsilon(\alpha, \cdot)$ is constant outside a countable union of proper subvarieties of $X$.

If $\theta \in \alpha$ is a Kähler form, we have by (2) and (3) that a necessary condition for the existence of a $\theta$-psh Green function with an isotropic pole at $p$ is

$$
\varepsilon(\alpha, p)=V_{\alpha}^{1 / n} \text {. }
$$

Since this fails to hold in general (see Proposition 3.1), one has to consider other singularities. Following ideas of Demailly [D5], we will show that local fundamental solutions of the Monge-Ampère operator have $\theta$-psh subextensions to $X$.

We will consider the slightly more general situation when the class $\alpha$ is represented by a smooth closed $(1,1)$ form $\theta \geq 0$ and $V_{\alpha}>0$. Recall that the unbounded locus $M(\varphi)$ of $\varphi \in P S H(X, \theta)$ is defined as the set of all points $p \in X$ such that $\varphi$ is unbounded in every neighborhood of $p$. We denote by $P S H^{-}(X, \theta)$ the set of $\theta$-psh functions $\varphi \leq 0$ on $X$. For $p \in X$, let $\mathcal{G}_{p}\left(V_{\alpha}\right)$ be the set of germs of functions $u$ at $p$ with the following properties: there exists an open set $U \subset X$ containing $p$ such 
that $u$ is psh on $U$ and locally bounded on $U \backslash\{p\}, u(p)=-\infty$, and $\left(d d^{c} u\right)^{n}=V_{\alpha} \delta_{p}$ as measures on $U$.

Theorem 1.4. Let $p \in X$ and $u \in \mathcal{G}_{p}\left(V_{\alpha}\right)$. There exists a unique function $g=$ $g_{u, p} \in P S H^{-}(X, \theta)$ such that

(i) $g \leq u+C$ holds near $p$, for some constant $C$.

(ii) If $\varphi \in P S H^{-}(X, \theta)$ and $\liminf _{q \rightarrow p} \varphi(q) / u(q) \geq 1$ then $\varphi \leq g$ on $X$. In addition, $g$ has the following properties:

(a) $\left(\theta+d d^{c} g\right)^{n}=0$ on the open set $X \backslash(M(g) \cup\{g=0\})$.

(b) If $p$ is an isolated point of $M(g)$ then $M(g)=\{p\}$ and $g$ is a $\theta$-psh Green function on $X$ with pole at $p$.

(c) The open set $D_{u, p}=\{g<0\}$ is connected.

It should be noted that the existence of a global $\theta$-psh function $\varphi$ subextending $u$ (i.e. such that $\varphi \leq u$ near $p$ ) is a nontrivial matter. We use Yau's solution in the spirit of [D5], [DP]. Producing the "best subextension" $g$ proceeds using a classical balayage procedure (see $[\mathrm{R}]$ for recent similar local extremal problems).

Proof. The uniqueness of a function with properties (i), (ii) is clear. Fix $U \subset X$ an open coordinate ball around $p$, so that $u$ is psh on $U$, locally bounded on $U \backslash\{p\}$ and $\left(d d^{c} u\right)^{n}=V_{\alpha} \delta_{p}$ as measures on $U$. We divide the proof in three steps.

Step 1. Using a mass concentration technique of Demailly [D5], we construct a function $\varphi \in P S H(X, \theta)$ so that $\varphi \leq u$ near $p$. Let $\omega_{0}$ be a Kähler form on $X$.

Let $W \subset \subset W^{\prime} \subset \subset U$ be open and connected, with $p \in W$, and let $\chi$ be a smooth function on $X$ with compact support in $W^{\prime}$, such that $0 \leq \chi \leq 1$ and $\chi=1$ on $W$. We may assume that $u \geq 0$ on $\partial W$. Let $\rho, \rho_{0}$ be negative smooth functions on $W^{\prime}$ with $d d^{c} \rho=\theta, d d^{c} \rho_{0}=\omega_{0}$.

Let $u_{j} \searrow u$ be a sequence of smooth psh functions on $W^{\prime}$ and let $\omega_{j}=\theta+j^{-1} \omega_{0}$. We define measures

$$
\mu_{j}=C_{j} \chi\left(d d^{c} u_{j}\right)^{n}
$$

where the constants $C_{j}>0$ are chosen so that $\mu_{j}(X)=\int_{X} \omega_{j}^{n}$. Note that $\mu_{j}$ has support in $W^{\prime}$, and $\left(d d^{c} u_{j}\right)^{n} \rightarrow V_{\alpha} \delta_{p}$ in the weak sense of measures on $W^{\prime}$. Hence

$$
\lim _{j \rightarrow \infty} \int \chi\left(d d^{c} u_{j}\right)^{n}=V_{\alpha} \chi(p)=V_{\alpha}, \text { so } \lim _{j \rightarrow \infty} C_{j}=1 .
$$

Yau's theorem (see $[Y]$, also [Ko1]) implies that there exist continuous functions $\varphi_{j} \in P S H\left(X, \omega_{j}\right)$ such that

$$
\left(\omega_{j}+d d^{c} \varphi_{j}\right)^{n}=\mu_{j}, \max _{X} \varphi_{j}=0 .
$$

By [GZ1, Proposition 1.7] we may assume after passing to a subsequence that $\left\{\varphi_{j}\right\}$ converges in $L^{1}(X)$ to a function $\varphi \in P S H(X, \theta)$. Moreover, by [Ho, Theorem 4.1.8] we have $\varphi=\left(\lim \sup _{j \rightarrow \infty} \varphi_{j}\right)^{\star}$ on $X$.

Choose a sequence $a_{j} \geq 1$ so that $a_{j}^{n} C_{j}>1$ and $a_{j} \rightarrow 1$. We have

$$
a_{j}\left(\varphi_{j}+\rho+j^{-1} \rho_{0}\right) \leq 0 \leq u_{j} \text { on } \partial W .
$$

On the other hand

$$
a_{j}^{n}\left(d d^{c}\left(\varphi_{j}+\rho+j^{-1} \rho_{0}\right)\right)^{n}=a_{j}^{n} C_{j} \chi\left(d d^{c} u_{j}\right)^{n} \geq\left(d d^{c} u_{j}\right)^{n}
$$


holds on $W$, as $\chi=1$ on $W$. The minimum principle of Bedford and Taylor [BT1, Theorem A] implies that $a_{j}\left(\varphi_{j}+\rho+j^{-1} \rho_{0}\right) \leq u_{j}$ on $W$. Letting $j \rightarrow \infty$ we obtain that $\varphi+\rho \leq u$ holds on $W$. This concludes Step 1 .

Step 2. We construct the function $g$ using an upper envelope method. Consider the family

$$
\mathcal{F}=\left\{\varphi \in P S H^{-}(X, \theta): \liminf _{q \rightarrow p} \frac{\varphi(q)}{u(q)} \geq 1\right\} .
$$

In the terminology of Rashkovskii, this is the family of negative $\theta$-psh functions whose relative type with respect to $u$ is at least 1 (see $[\mathrm{R}]$ ).

By Step $1, \mathcal{F} \neq \emptyset$. If $g=\sup \{\varphi: \varphi \in \mathcal{F}\}$, then the upper semicontinuous regularization $g^{\star} \in P S H^{-}(X, \theta)$. We will show that $g^{\star} \leq u+C$ holds near $p$ for some constant $C$. This implies that $g=g^{\star} \in \mathcal{F}$, so $g$ verifies properties $(i),(i i)$.

We can find $M>0$ such that the connected component $D$ of $\{u<-M\}$ which contains $p$ is relatively compact in $U$. Let $\rho<0$ be a smooth function on $U$ so that $d d^{c} \rho=\theta$. Fix $\varphi \in \mathcal{F}$. There exists a sequence of relatively compact domains $D_{j} \subset D, j>0$, with the following properties:

$$
D_{j+1} \subset D_{j}, \bigcap_{j>0} D_{j}=\{p\}, \varphi(q) \leq\left(1-j^{-1}\right) u(q) \text { for } q \in \bar{D}_{j} .
$$

We have $\rho+\varphi \leq 0 \leq\left(1-j^{-1}\right)(u+M)$ on $\partial D$, and clearly $\rho+\varphi \leq\left(1-j^{-1}\right)(u+M)$ on $\partial D_{j}$. Since the psh function $u$ is maximal on $U \backslash\{p\}$, it follows that the last inequality holds on $D \backslash D_{j}$. As $j \rightarrow \infty$ we see that $\rho+\varphi \leq u+M$ on $D$. Since $\varphi \in \mathcal{F}$ was arbitrary, this implies that $g^{\star} \leq u+C$ on $D$, where $C=M-\min _{D} \rho$.

Step 3. We prove the remaining properties of $g$.

(a) Note that $M(g)$ is closed and since $g \leq 0$ is upper semicontinuous the set $\{g=0\}$ is closed. Let $q \in X \backslash(M(g) \cup\{g=0\})$ and let $\rho$ be a smooth function in a neighborhood of $q$ such that $d d^{c} \rho=\theta$ and $\rho(q)=0$. We can find $\varepsilon>0$ and a small neighborhood $G$ of $q$ such that $G \subset X \backslash(M(g) \cup\{g=0\})$ and $g<-\varepsilon,|\rho|<\varepsilon / 2$ on $G$. Let $W$ be a relatively compact open subset of $G$ and $v$ be psh on $W$ so that $v^{\star} \leq \rho+g$ on $\partial W$. The function

$$
\varphi=g \text { on } X \backslash W, \quad \varphi=\max \{\rho+g, v\}-\rho \text { on } W,
$$

is $\theta$-psh and $\varphi \leq 0$ on $X$. Since $\varphi=g$ in a neighborhood of $p$, we conclude that $\varphi \in \mathcal{F}$, hence $v \leq \rho+g$ on $W$. This shows that the psh function $\rho+g$ is maximal on $G$. By [BT2], $\left(\theta+d d^{c} g\right)^{n}=0$ in $G$, and hence on $X \backslash(M(g) \cup\{g=0\})$.

(b) If $p \in M(g)$ is isolated, there exists a closed ball $K$ centered at $p$ so that $K \cap M(g)=\{p\}$. Hence $g$ is bounded below on $\partial K$. It follows that if $C>0$ is large enough the function $\varphi$ defined by $\varphi=g$ on $K, \varphi=\max \{g,-C\}$ on $X \backslash K$, is $\theta$-psh and $\varphi \in \mathcal{F}$. Thus $\varphi \leq g$, so $M(g)=\{p\}$. By $(i)$ and [D4], $\left(\theta+d d^{c} g\right)^{n}(\{p\}) \geq$ $\left(d d^{c} u\right)^{n}(\{p\})=V_{\alpha}$. Mass considerations imply that $g$ is a $\theta$-psh Green function.

(c) Suppose that there exists a connected component $W$ of $D_{u, p}$ not containing $p$. The function $\varphi$ defined by $\varphi=g$ on $X \backslash W$ and $\varphi=0$ on $W$, verifies $\varphi \in \mathcal{F}$, so $\varphi \leq g$. This contradicts our assumption that $g<0$ on $W$, so $D_{u, p}$ is connected.

The following theorem produces Green functions with several poles. Its proof is a straightforward adaptation of the proof of Theorem 1.4. 
Theorem 1.5. For $1 \leq j \leq k$, let $p_{j} \in X, u_{j} \in \mathcal{G}_{p_{j}}\left(V_{\alpha}\right)$, and $m_{j}>0$ with $\sum_{j=1}^{k} m_{j}=1$. There exists a unique function $g \in \operatorname{PSH}^{-}(X, \theta)$ such that

(i) $g \leq m_{j}^{1 / n} u_{j}+C$ holds near each $p_{j}$, for some constant $C$.

(ii) If $\varphi \in P S H^{-}(X, \theta)$ and for each $j$, $\liminf _{q \rightarrow p_{j}} \varphi(q) / u_{j}(q) \geq m_{j}^{1 / n}$, then $\varphi \leq g$ on $X$.

Moreover, we have $\left(\theta+d d^{c} g\right)^{n}=0$ on $X \backslash(M(g) \cup\{g=0\})$. If all $p_{j}$ are isolated points of $M(g)$ then $g$ is a $\theta$-psh Green function with poles at $p_{1}, \ldots, p_{k}$.

It is an intricate problem to decide whether there always exist local models $u$ at $p \in X$ such that $g_{u, p}$ is a Green function. As an alternate approach, we introduce a partial Green function associated to an isotropic singularity.

Proposition 1.6. Let $\theta \in \alpha$ be a Kähler form, let $p \in X$ and $0<\gamma<\varepsilon(\alpha, p)$. There exists a unique function $\psi_{\gamma, p} \in P S H^{-}(X, \theta)$ so that $\nu\left(\psi_{\gamma, p}, p\right)=\gamma$ and with the property that if $\varphi \in P S H^{-}(X, \theta)$ and $\nu(\varphi, p) \geq \gamma$ then $\varphi \leq \psi_{\gamma, p}$. Moreover,

$$
\left\|\psi_{\gamma, p}-\gamma \log \operatorname{dist}(\cdot, p)\right\|_{L^{\infty}(X)}<+\infty,\left(\theta+d d^{c} \psi_{\gamma, p}\right)^{n}=\gamma^{n} \delta_{p}+\mu_{\gamma, p},
$$

where $\mu_{\gamma, p}$ is a positive measure supported on the compact $\left\{\psi_{\gamma, p}=0\right\}$.

Proof. The uniqueness of $\psi_{\gamma, p}$ is clear. Let us fix a biholomorphic map $f: B \rightarrow U$ from the unit ball $B \subset \mathbb{C}^{n}$ onto a neighborhood $U$ of $p$, with $f(0)=p$. Let $\rho<0$ be a smooth function on $U$ with $d d^{c} \rho=\theta$.

By (3) there exists $\psi \in P S H^{-}(X, \theta)$ so that $\psi=\gamma \log \operatorname{dist}(\cdot, p)+O(1)$. Let

$$
\psi_{\gamma, p}(q)=\sup \left\{\varphi(q): \varphi \in P S H^{-}(X, \theta), \nu(\varphi, p) \geq \gamma\right\} .
$$

For such $\varphi$, we have $(\rho+\varphi)(f(z)) \leq \gamma \log \|z\|$ on $B$. This implies $\psi_{\gamma, p}^{\star} \in P S H^{-}(X, \theta)$ and $\nu\left(\psi_{\gamma, p}^{\star}, p\right) \geq \gamma$. Thus $\psi_{\gamma, p}=\psi_{\gamma, p}^{\star}$. Since $\psi \leq \psi_{\gamma, p}$, it follows that $\nu\left(\psi_{\gamma, p}, p\right)=\gamma$ and the function $\psi_{\gamma, p}-\gamma \log \operatorname{dist}(\cdot, p)$ is bounded on $X$.

Arguing as in the proof of Theorem $1.4(a)$ we show that $\left(\theta+d d^{c} \psi_{\gamma, p}\right)^{n}=0$ in $\left\{\psi_{\gamma, p}<0\right\} \backslash\{p\}$. By [D4], $\left(\theta+d d^{c} \psi_{\gamma, p}\right)^{n}(\{p\})=\gamma^{n}$, and the proof is complete.

We refer to $[R]$ for similar extremal problems on domains in $\mathbb{C}^{n}$. In the following sections, we are going to compute the functions $\nu, \varepsilon$ and $g_{u, p}, \psi_{\nu, p}$ in a number of interesting cases.

\section{Green functions on $\mathbb{P}^{n}$}

Let $\left[z_{0}: \ldots: z_{n}\right]$ be homogeneous coordinates on $\mathbb{P}^{n}$ and $\pi_{n}: \mathbb{C}^{n+1} \backslash\{0\} \rightarrow \mathbb{P}^{n}$ be the standard projection. Let $\alpha_{n}=\left\{\omega_{n}\right\}$, where $\omega_{n}$ is the Fubini-Study form, so $\pi_{n}^{\star} \omega_{n}=d d^{c} \log \|z\|$ and $\operatorname{Vol}\left(\alpha_{n}\right)=1$.

\subsection{Maximal Lelong number.}

Proposition 2.1. We have $\nu\left(\alpha_{n}, x\right)=\varepsilon\left(\alpha_{n}, x\right)=1$ for all $x \in \mathbb{P}^{n}$. If $T \in \mathcal{P}\left(\alpha_{n}\right)$ and $\nu(T, x)=1$ then $T=\wp_{x}^{\star} S$, where $\wp_{x}: \mathbb{P}^{n} \rightarrow \mathbb{P}^{n-1}$ is the projection with center $x$ onto a hyperplane $\mathbb{P}^{n-1} \not \supset x$ and $S \in \mathcal{P}\left(\alpha_{n-1}\right)$. Moreover, the following are equivalent:

(i) the potentials of $T$ have isotropic pole at $x$ with Lelong number 1.

(ii) $T$ has locally bounded potentials on $\mathbb{P}^{n} \backslash\{x\}$.

(iii) $S$ has bounded potentials. 
Proof. Let $\pi: X \rightarrow \mathbb{P}^{n}$ denote the blow up of $\mathbb{P}^{n}$ at $x$, and let $E$ be the exceptional divisor. The map $\Phi=\wp_{x} \circ \pi: X \rightarrow \mathbb{P}^{n-1}$ is a holomorphic fibration, whose fibers are the projective lines through $x$. Moreover, $\pi^{\star} \alpha_{n}-E=\Phi^{\star} \alpha_{n-1}$.

If $\nu(T, x)=1$ then $\widetilde{T}=\pi^{\star} T-[E]$ is a positive closed $(1,1)$-current on $X$ in the cohomology class $\Phi^{\star} \alpha_{n-1}$. It follows that $\widetilde{T}=\Phi^{\star} S$ for some $S \in \mathcal{P}\left(\alpha_{n-1}\right)$, hence $T=\wp_{x}^{\star} S$. The potentials of $T$ have isotropic pole at $x$ with Lelong number 1 if and only if $\widetilde{T}$ has bounded potentials, hence if and only if $S$ has bounded potentials.

It is well known that currents in $\mathcal{P}\left(\alpha_{n}\right)$ have Lelong number at most 1 at each point $x$. The above construction shows that $\nu\left(\alpha_{n}, x\right)=\varepsilon\left(\alpha_{n}, x\right)=1$.

We now explore further the geometry of sublevel sets of high Lelong numbers, in the spirit of [Co2]. For $c>0$ and $T \in \mathcal{P}\left(\alpha_{n}\right)$ a theorem of Siu [Si] states that

$$
E_{c}(T):=\left\{x \in \mathbb{P}^{n}: \nu(T, x) \geq c\right\}
$$

is an algebraic subset of dimension at most $n-1$. We also consider the set

$$
E_{c}^{+}(T):=\left\{x \in \mathbb{P}^{n}: \nu(T, x)>c\right\} .
$$

Proposition 2.2. The set $E_{n /(n+1)}^{+}(T)$ is contained in a hyperplane of $\mathbb{P}^{n}$.

Proof. Let $T=\omega_{n}+d d^{c} \varphi$ and set $E_{c}(\varphi)=E_{c}(T)$ and $E_{c}^{+}(\varphi)=E_{c}^{+}(T)$. The proof is by induction on $n$. If $n=1, T$ is a probability measure, $\nu(T, p)=T(\{p\})$, so $E_{1 / 2}^{+}(T)$ contains at most one point.

Let $c_{n}=n /(n+1)$. If $n \geq 2$ we assume for a contradiction that $E_{c_{n}}^{+}(\varphi)$ contains the points $q, p_{1}, \ldots, p_{n}$ in general position. Let $H$ be the hyperplane determined by $p_{1}, \ldots, p_{n}$, so $q \notin H$. By a theorem of Siu [Si], $T=c[H]+R$, where $0 \leq c \leq 1$ and $R \in \mathcal{P}\left((1-c) \alpha_{n}\right)$ has generic Lelong number 0 along $H$. Thus

$$
c_{n}<\nu(\varphi, q)=\nu(R, q) \leq 1-c, \nu\left(R, p_{j}\right)=\nu\left(\varphi, p_{j}\right)-c>c_{n}-c, 1 \leq j \leq n .
$$

Consider the current $S=R /(1-c)=\omega_{n}+d d^{c} \psi \in \mathcal{P}\left(\alpha_{n}\right)$. Since $c<1-c_{n}$,

$$
\nu\left(\psi, p_{j}\right)>\frac{c_{n}-c}{1-c}>\frac{2 c_{n}-1}{c_{n}}=c_{n-1}, 1 \leq j \leq n .
$$

By [D3, Proposition 3.7], there exist $\epsilon_{k} \searrow 0$ and currents $S_{k}=\left(1+\epsilon_{k}\right) \omega_{n}+d d^{c} \psi_{k} \geq 0$, where $\psi_{k}$ have analytic singularities, such that $S_{k} \rightarrow S$ and $0 \leq \nu(\psi, p)-\nu\left(\psi_{k}, p\right) \leq$ $\epsilon_{k}$ for all $p \in \mathbb{P}^{n}$. Since $S$ does not charge $H$, it follows that $\psi_{k} \not \equiv-\infty$ on $H \equiv \mathbb{P}^{n-1}$. Hence $\left.\psi_{k}\right|_{H} \in P S H\left(\mathbb{P}^{n-1}, \omega_{n-1}\right)$ and

$$
\nu\left(\left.\psi_{k}\right|_{H}, p_{j}\right) \geq \nu\left(\psi_{k}, p_{j}\right)>c_{n-1}, 1 \leq j \leq n,
$$

for $k$ sufficiently large. This yields a contradiction, since by our induction hypothesis the set $E_{(n-1) / n}^{+}\left(\left.\psi_{k}\right|_{H}\right)$ is contained in a hyperplane of $\mathbb{P}^{n-1}$.

The value $n /(n+1)$ in the previous theorem is sharp. Indeed, let $S$ be a set of $n+1$ points $p_{j} \in \mathbb{P}^{n}$ in general position, and let $\left[H_{j}\right]$ be the current of integration along the hyperplane $H_{j}$ determined by $S \backslash\left\{p_{j}\right\}$. If $T=\left(\left[H_{1}\right]+\ldots+\left[H_{n+1}\right]\right) /(n+1)$ then the set $E_{n /(n+1)}(T)=S$ is not contained in any hyperplane.

We are now in position to make the result of Proposition 2.1 more precise, by giving a characterization of the currents $T$ for which $E_{1}(T) \neq \emptyset$. 
Proposition 2.3. If $T \in \mathcal{P}\left(\alpha_{n}\right)$ and $E_{1}(T) \neq \emptyset$ then $E_{1}(T)$ is a k-dimensional linear subspace of $\mathbb{P}^{n}$ for some integer $0 \leq k \leq n-1$. Let $\wp$ denote the projection with center $E_{1}(T)$ onto a linear subspace $L \equiv \mathbb{P}^{n-k-1}$ such that $L \cap E_{1}(T)=\emptyset$. Then $T=\wp^{\star} S$ for a unique current $S \in \mathcal{P}\left(\alpha_{n-k-1}\right)$, and $E_{1}(S)=\emptyset$.

Proof. Let $T=\omega_{n}+d d^{c} \varphi$ and $k \geq 0$ be the largest integer for which there exist $k+1$ points $p_{0}, \ldots, p_{k} \in E_{1}(T)$ in general position (i.e. not contained in a $(k-1)$ dimensional subspace). Proposition 2.2 implies $k \leq n-1$. Using an automorphism of $\mathbb{P}^{n}$, we may assume $p_{0}=[1: 0: \ldots: 0], p_{1}=[0: 1: \ldots: 0]$, and so on. Consider the projection $f_{0}$ of $\mathbb{P}^{n}$ with center $p_{0}$ onto the hyperplane $\mathbb{P}^{n-1} \equiv\left\{z_{0}=0\right\}$. Proposition 2.1 shows that $\varphi=u+h_{0} \circ f_{0}$, where $h_{0} \in P S H\left(\mathbb{P}^{n-1}, \omega_{n-1}\right)$ and

$$
u\left(\left[z_{0}: \ldots: z_{n}\right]\right)=\frac{1}{2} \log \frac{\left|z_{1}\right|^{2}+\ldots+\left|z_{n}\right|^{2}}{\left|z_{0}\right|^{2}+\ldots+\left|z_{n}\right|^{2}} .
$$

It follows that $f_{0}\left(p_{j}\right) \in E_{1}\left(h_{0}\right), j=1, \ldots, k$, and Proposition 2.1 can be applied to $h_{0}$ and the point $f_{0}\left(p_{1}\right)$. Continuing like this we get

$$
\varphi\left(\left[z_{0}: \ldots: z_{n}\right]\right)=\frac{1}{2} \log \frac{\left|z_{k+1}\right|^{2}+\ldots+\left|z_{n}\right|^{2}}{\left|z_{0}\right|^{2}+\ldots+\left|z_{n}\right|^{2}}+h\left(\left[z_{k+1}: \ldots: z_{n}\right]\right),
$$

with $h \in P S H\left(\mathbb{P}^{n-k-1}, \omega_{n-k-1}\right)$. The definition of $k$ implies $E_{1}(h)=\emptyset$, so $E_{1}(\varphi)=$ $\left\{z_{k+1}=\ldots=z_{n}=0\right\}$.

\subsection{Green functions.}

2.2.1. Green functions with one pole. It follows from Proposition 2.1 that if $T=$ $\wp_{x}^{\star} S$, where $S \in \mathcal{P}\left(\alpha_{n-1}\right)$ has bounded potentials and $\wp_{x}: \mathbb{P}^{n} \rightarrow \mathbb{P}^{n-1}$ is the projection from $x$, then $T=\omega_{n}+d d^{c} g$ with $g=g_{S, x} \in P S H\left(\mathbb{P}^{n}, \omega_{n}\right) \cap L_{l o c}^{\infty}\left(\mathbb{P}^{n} \backslash\{x\}\right)$, $g$ has an isotropic pole at $x$ with Lelong number 1 and

$$
\left(\omega_{n}+d d^{c} g\right)^{n}=\delta_{x} .
$$

Conversely, any $\omega_{n}$-psh Green function $g$ with pole at $x$ and maximal Lelong number $\nu(g, x)=1$ is of this form, and in particular it must have an isotropic pole at $x$. Observe that the set of such functions is large.

2.2.2. Multipole Green functions. We push further the result of Proposition 2.1 and study multipole Green functions which arise naturally from rational maps.

Let $f: \mathbb{P}^{n} \rightarrow \mathbb{P}^{n-1}, f=\left[P_{1}: \ldots: P_{n}\right]$, be a rational map with finite indeterminacy set $I_{f}$, where $P_{j}$ are homogeneous polynomials of degree $d$ on $\mathbb{C}^{n+1}$. Then $f$ determines an $\omega_{n}$-psh Green function,

$$
g_{f}\left(\pi_{n}(z)\right)=d^{-1} \log \|F(z)\|-\log \|z\|, z \in \mathbb{C}^{n+1} \backslash\{0\},
$$

where $F: \mathbb{C}^{n+1} \rightarrow \mathbb{C}^{n}, F(z)=\left(P_{1}(z), \ldots, P_{n}(z)\right)$. The function $g_{f}$ is continuous, $I_{f}=\left\{g_{f}=-\infty\right\}$, and $g_{f}$ has an isolated pole at each point of $I_{f}$. Moreover, $g_{f}$ verifies the Monge-Ampère equation

$$
\left(\omega_{n}+d d^{c} g_{f}\right)^{n}=\sum_{p \in I_{f}} m_{p} \delta_{p}, \text { where } m_{p}>0, m_{p} \in \mathbb{Q}, \sum_{p \in I_{f}} m_{p}=1 .
$$

Our next result shows that this function has an extremal property (see [Co1] for a similar characterization of classes of pluricomplex Green functions on $\mathbb{C}^{n}$ ): 
Theorem 2.4. If $\varphi \in P S H\left(\mathbb{P}^{n}, \omega_{n}\right)$ and $\varphi \leq g_{f}$, then there exists a unique function $h \in P S H\left(\mathbb{P}^{n-1}, \omega_{n-1}\right)$ such that $\varphi=g_{f}+d^{-1} h \circ f$. Conversely, any such function $\varphi$ is $\omega_{n}$-psh. We have that $\varphi$ is locally bounded on $\mathbb{P}^{n} \backslash I_{f}$ if and only if $h$ is bounded. In this case, $\varphi$ satisfies

$$
\left(\omega_{n}+d d^{c} \varphi\right)^{n}=\sum_{p \in I_{f}} m_{p} \delta_{p}
$$

Proof. Since the indeterminacy set $I_{f}$ is finite, we can find a hyperplane $H$ which does not intersect $I_{f}$. Let $L$ be a linear polynomial defining $H$, and let $P_{0}=L^{d}$. The map $\hat{f}=\left[P_{0}: P_{1}: \ldots: P_{n}\right]: \mathbb{P}^{n} \rightarrow \mathbb{P}^{n}$ is holomorphic and $f=\wp \circ \hat{f}$, where

$$
\wp: \mathbb{P}^{n} \rightarrow \mathbb{P}^{n-1}, \wp\left(\left[z_{0}: z_{1}: \ldots: z_{n}\right]\right)=\left[z_{1}: \ldots: z_{n}\right],
$$

is the projection with center $[1: 0: \ldots: 0]$.

For every $p \in \mathbb{P}^{n-1}$ the fiber $X_{p}:=f^{-1}(p)=\hat{f}^{-1}\left(\wp^{-1}(p)\right)$ is one-dimensional and is connected by [FH, Proposition 1], since $\wp^{-1}(p)$ is a line in $\mathbb{P}^{n}$. This implies in particular the uniqueness of $h$.

Fix now an arbitrary $p \in \mathbb{P}^{n-1}$, and let us assume $p=\left[a_{1}: \ldots: a_{n-1}: 1\right]$. Then $X_{p}$ is defined by the equations $P_{j}=a_{j} P_{n}$. Let $q=\left[b_{0}: \ldots: b_{n}\right]$ be a point in $X_{p} \backslash I_{f}$. We assume that $b_{0}=1$. Then $q$ has a neighborhood where $P_{n}\left(1, z_{1}, \ldots, z_{n}\right) \neq 0$. So, for some constant $c$, we have $\log \|F\|=\log \left|P_{n}\right|+c$ in this neighborhood. It follows that $\varphi-g_{f}$ is psh in some open set which contains $X_{p} \backslash I_{f}$. Since $\varphi-g_{f} \leq 0$ and $I_{f}$ is a finite set, $\varphi-g_{f}$ extends to a subharmonic function on $X_{p}$. But $X_{p}$ is compact and connected, so $\varphi-g_{f}$ is constant on $X_{p}$. We conclude that $\varphi=g_{f}+(h \circ f) / d$, for some function $h$ on $\mathbb{P}^{n-1}$. Since $\varphi \leq g_{f}$ and $g_{f}$ is continuous, it follows easily that $h$ is upper semicontinuous.

We now show that $h \in P S H\left(\mathbb{P}^{n-1}, \omega_{n-1}\right)$. By using an automorphisms of $\mathbb{P}^{n}$ we may assume that the hyperplane $H=\left\{z_{0}=0\right\}$ does not intersect $I_{f}$. We claim that the map $F^{\prime}: \mathbb{C}^{n} \rightarrow \mathbb{C}^{n}, F^{\prime}\left(z^{\prime}\right)=F\left(1, z^{\prime}\right)$, is proper. Indeed, if $P_{j}^{d}\left(z^{\prime}\right)$ is the homogeneous part of degree $d$ of $P_{j}\left(1, z^{\prime}\right)$, then $P_{j}^{d}\left(z^{\prime}\right), j=1, \ldots, n$, have no common zeros except at 0 . The homogeneity of $P_{j}^{d}$ yields

$$
\sum_{j=1}^{n}\left|P_{j}^{d}\left(z^{\prime}\right)\right|^{2} \geq M\left\|z^{\prime}\right\|^{2 d},
$$

for some constant $M>0$, which implies that $F^{\prime}$ is proper. The function

$$
u\left(z^{\prime}\right)=\varphi\left(\left[1: z^{\prime}\right]\right)+\log \sqrt{1+\left\|z^{\prime}\right\|^{2}}=\frac{1}{d} \log \left\|F^{\prime}\left(z^{\prime}\right)\right\|+\frac{1}{d} h \circ \pi_{n-1}\left(F^{\prime}\left(z^{\prime}\right)\right)
$$

is psh on $\mathbb{C}^{n}$. Since $F^{\prime}$ is proper, the function

$$
v(w)=d \max \left\{u\left(z^{\prime}\right): F^{\prime}\left(z^{\prime}\right)=w\right\}=\log \|w\|+h \circ \pi_{n-1}(w)
$$

is psh on $\mathbb{C}^{n}$. This proves that $h \in P S H\left(\mathbb{P}^{n-1}, \omega_{n-1}\right)$.

For the converse, note that

$$
\omega_{n}+d d^{c}\left(g_{f}+(h \circ f) / d\right)=d^{-1} f^{\star}\left(\omega_{n-1}+d d^{c} h\right) \geq 0,
$$

so $g_{f}+(h \circ f) / d$ is $\omega_{n}$-psh.

Finally, it is clear that $\varphi \in L_{\text {loc }}^{\infty}\left(\mathbb{P}^{n} \backslash I_{f}\right)$ if and only if $h$ is bounded. Then we infer by [D4] that $m_{p}=\left(\omega_{n}+d d^{c} g_{f}\right)^{n}(\{p\})=\left(\omega_{n}+d d^{c} \varphi\right)^{n}(\{p\})$. The conclusion follows since $\sum_{p \in I_{f}} m_{p}=1$. 
Note that Proposition 2.1 follows from Theorem 2.4 applied to rational maps of degree $d=1$. We will see in section 3.2 that Green functions determined by certain rational maps $f: \mathbb{P}^{2} \rightarrow \mathbb{P}^{1}$ with three points of indeterminacy provide rich classes of examples of Green functions with one pole on $\mathbb{P}^{1} \times \mathbb{P}^{1}$ (see Example 3.5).

Example 2.5. An important particular case of Theorem 2.4 is the one of rational functions $f: \mathbb{P}^{2} \rightarrow \mathbb{P}^{1}, f=\left[P_{1}: P_{2}\right]$, where $P_{j}$ are homogeneous polynomials of degree $d$ whose common zero set $I_{f}$ consists of $d^{2}$ distinct points of $\mathbb{P}^{2}$. Then $g_{f}$ is a $\omega_{2}$-psh Green function with $d^{2}$ isotropic poles and Lelong number 1/d at each pole. If $d=2$ we observe that any set of four points in general position is the complete intersection of two conics, hence it can be realized as the indeterminacy set $I_{f}$ for a rational map $f$ of degree $d=2$ as described above. It follows that the $\omega_{2}$-psh Green functions with four isotropic poles are described by Theorem 2.4. However, if $d \geq 3$ a set of $d^{2}$ points of $\mathbb{P}^{2}$ in general position is not the complete intersection of two curves of degree $d$ (in fact when $d \geq 4$, there is no curve of degree $d$ passing through $d^{2}$ points in general position). So the Green functions $g_{f}$ with $d^{2}$ isotropic poles, $d \geq 3$, only exist for very special sets of poles.

2.2.3. Partial Green functions. We compute here in the case of $\left(\mathbb{P}^{n}, \omega_{n}\right)$ the functions $\psi_{\nu, p}$ constructed in Proposition 1.6. Assume without loss of generality that $p=0 \in \mathbb{C}^{n}$. For $\nu<1$ define $R_{\nu}, C_{\nu}$ by

$$
R_{\nu}=[\nu /(1-\nu)]^{1 / 2}, \nu \log R_{\nu}+C_{\nu}=\log \sqrt{1+R_{\nu}^{2}} .
$$

For $z \in \mathbb{C}^{n}$ let

$$
V(z)=\left\{\begin{array}{l}
\nu \log \|z\|+C_{\nu},\|z\| \leq R_{\nu} \\
\log \sqrt{1+\|z\|^{2}},\|z\| \geq R_{\nu} .
\end{array}\right.
$$

Proposition 2.6. For $\nu<1$ and $z \in \mathbb{C}^{n}$ we have $\psi_{\nu, p}(z)=V(z)-\log \sqrt{1+\|z\|^{2}}$.

Proof. Note that $\psi_{\nu, p}(z)=W(z)-\log \sqrt{1+\|z\|^{2}}$, where

$$
W(z)=\sup \left\{v(z): v \in P S H\left(\mathbb{C}^{n}\right), v \leq \log \sqrt{1+\|\cdot\|^{2}}, \nu(v, 0) \geq \nu\right\} .
$$

Since $\max _{\|z\|=r} v(z)$ is a convex increasing function of $\log r$, and since $x=\log R_{\nu}$ is the solution of the equation $\frac{d}{d x} \log \sqrt{1+e^{2 x}}=\nu$, it follows that $W=V$.

Letting $\nu \nearrow 1$ it follows that $\psi_{1, p}(z)=\log \left(\|z\| / \sqrt{1+\|z\|^{2}}\right), z \in \mathbb{C}^{n}$, is the Green function constructed in Theorem 1.4 for $u(z)=\log \|z\|$.

2.2.4. Dynamical Green functions. We now consider the problem of constructing Green functions on $\mathbb{P}^{2}$ with one pole at $p$ and Lelong number at $p$ less than 1 . Let $\omega=\omega_{2}$, let $[t: x: y]$ denote the homogeneous coordinates on $\mathbb{P}^{2}$, and identify $z=(x, y) \in \mathbb{C}^{2}$ to $[1: x: y]$. Simple examples can be obtained by considering a smooth curve with a flex at $p$, i.e. the tangent line at $p$ does not intersect the curve at any other points. More generally, for integers $1 \leq k<n$, the function

$$
g([t: x: y])=\frac{1}{2 n} \log \left(\left|y^{k} t^{n-k}-x^{n}\right|^{2}+\left|y^{n}\right|^{2}\right)-\frac{1}{2} \log \left(|t|^{2}+|x|^{2}+|y|^{2}\right)
$$

is $\omega$-psh and smooth away from $p=0 \in \mathbb{C}^{2}, \nu(g, p)=k / n$ and $\left(\omega+d d^{c} g\right)^{2}=\delta_{p}$.

We describe next more elaborate constructions using complex dynamics. Let $h: \mathbb{C}^{2} \rightarrow \mathbb{C}^{2}$ be a polynomial mapping of algebraic degree $\lambda>1$. Then $h$ extends 
to a rational self-map of $\mathbb{P}^{2}$, denoted again by $h$, with finite indeterminacy set $I \subset\{t=0\}$. We call $h$ weakly regular if $h$ maps $\{t=0\} \backslash I$ to a point $Z \notin I$ (see [GS]). Such $h$ is algebraically stable $\left(\operatorname{deg} h^{n}=\lambda^{n}\right)$. It was shown in [S] that the currents $\lambda^{-n}\left(h^{n}\right)^{\star} \omega$ converge weakly to an invariant positive closed current $T=T_{h}$ on $\mathbb{P}^{2}, T=\omega+d d^{c} g$. We call $T$ the dynamical Green current and $g$ a dynamical Green function of $h$. By [GS, Theorem 2.2], $g$ is continuous on $\mathbb{P}^{2} \backslash I, T \wedge T$ is supported on $I$, so $g$ is a $\omega$-psh Green function with poles in $I$.

If $|I|=1$ then $T \wedge T=\delta_{I}$. Our goal is to compute the Lelong number $\nu(T, I)$.

Proposition 2.7. Let $h$ be a weakly regular polynomial endomorphism of $\mathbb{C}^{2}$ of degree $\lambda>1$, with $|I|=1$, and such that

$$
\operatorname{dist}(h(p), I) \geq C \operatorname{dist}(p, I)^{\delta}, p \in \mathbb{P}^{2} \backslash\{I\},
$$

for constants $0<C<1,1<\delta<\lambda$. Then $\nu\left(\lambda^{-n}\left(h^{n}\right)^{\star} \omega, I\right) \nearrow \nu(T, I)$ as $n \nearrow \infty$.

Proof. If $\lambda^{-1} h^{\star} \omega=\omega+d d^{c} \psi$, where $\psi \leq 0$ is $\omega$-psh, then by [G, Theorem 2.1]

$$
T_{n}:=\lambda^{-n}\left(h^{n}\right)^{\star} \omega=\omega+d d^{c} g_{n}, g_{n}=\sum_{j=0}^{n-1} \lambda^{-j} \psi \circ h^{j} \searrow g=\sum_{j=0}^{\infty} \lambda^{-j} \psi \circ h^{j},
$$

and $T=\omega+d d^{c} g$. Hence $\left\{\nu\left(T_{n}, I\right)\right\}$ is increasing and $\nu\left(T_{n}, I\right) \leq \nu(T, I)$.

It follows from (5) that there is $C^{\prime}>0$ so that for every $n$ and $p \in \mathbb{P}^{2} \backslash\{I\}$

$$
\operatorname{dist}\left(h^{n}(p), I\right) \geq\left(C^{\prime} \operatorname{dist}(p, I)\right)^{\delta^{n}} .
$$

Note that the function $\psi$ is smooth except at $I$, and $\psi \geq \gamma \log \operatorname{dist}(\cdot, I)-M$ holds on $\mathbb{P}^{2}$ for some constants $\gamma, M>0$. Writing $g=g_{n}+\rho_{n}$, we deduce that

$$
\rho_{n}(p) \geq \sum_{j=n}^{\infty} \lambda^{-j}\left(\gamma \log \operatorname{dist}\left(h^{j}(p), I\right)-M\right) \geq \gamma^{\prime}(\delta / \lambda)^{n} \log \operatorname{dist}(p, I)-\epsilon_{n},
$$

with some $\gamma^{\prime}>0$ and $\epsilon_{n} \rightarrow 0$. Thus $\nu\left(T_{n}, I\right) \leq \nu(T, I) \leq \nu\left(T_{n}, I\right)+\gamma^{\prime}(\delta / \lambda)^{n}$.

Note that (5) holds for Hénon maps $h(x, y)=(P(x)+a y, x), \operatorname{deg} P=\lambda$, with $\delta=1$, since $I=[0: 0: 1]$ is an attracting fixed point for $h^{-1}$. However, the map $h(x, y)=\left(x^{\lambda}-y^{\lambda-1}, y^{\lambda-1}\right)$ shows that (5) does not hold for $\delta<\lambda$.

Proposition 2.8. Let $h(x, y)=\left(x^{\lambda}+y^{\mu}, x\right)$, where $\lambda>\mu \geq 1$ are integers, so $I=[0: 0: 1]$. The Green current $T$ of $h$ verifies $T \wedge T=\delta_{I}, \nu(T, I)=(\lambda-\mu) / \lambda$.

Proof. We show first that (5) holds with $\delta=\lambda-1$. Note that $h$ is weakly regular and in local coordinates $(t, x)$ near $I$ we have

$$
h(t, x)=\left(\frac{t}{x}, \frac{x^{\lambda}+t^{\lambda-\mu}}{x t^{\lambda-1}}\right) .
$$

It is enough to prove (5) for $p=(t, x)$ with $0<|x|,|t|<1$. If $|t| \geq|x|$, or if $\left|x^{\lambda}+t^{\lambda-\mu}\right| \geq\left|x t^{\lambda-1}\right|$, then $\|h(t, x)\| \geq 1$ and the estimate follows. Otherwise, we have $|t|<|x|<1$ and $\left|x^{\lambda}+t^{\lambda-\mu}\right|<\left|x t^{\lambda-1}\right|$, so $|x|^{\lambda}<2|t|^{\lambda-\mu}$. Therefore

$$
\|h(t, x)\| \geq \frac{|t|}{|x|} \geq C|x|^{\mu /(\lambda-\mu)} \geq C|x|^{\lambda-1} \geq C^{\prime} \operatorname{dist}(p, I)^{\lambda-1} .
$$


Next we compute $\nu_{n}:=\nu\left(\lambda^{-n}\left(h^{n}\right)^{\star} \omega, I\right)$. Let $h^{n}\left([t: x: y]=\left[t^{\lambda^{n}}: p_{n}(t, x, y)\right.\right.$ : $\left.q_{n}(t, x, y)\right]$, where $p_{n}, q_{n}$ are homogeneous polynomials of degree $\lambda^{n}$, and

$$
v_{n}(t, x)=\log \left(|t|^{2 \lambda^{n}}+\left|p_{n}(t, x, 1)\right|^{2}+\left|q_{n}(t, x, 1)\right|^{2}\right)^{1 / 2} .
$$

It follows by induction that $\nu\left(v_{n}, 0\right)=\lambda^{n}-\max \left\{\operatorname{deg}_{y} p_{n}, \operatorname{deg}_{y} q_{n}\right\}=\lambda^{n}-\mu \lambda^{n-1}$, where $\operatorname{deg}_{y} p_{n}$ denotes the degree in $y$ of $p_{n}$. Hence $\nu_{n}=(\lambda-\mu) / \lambda=\nu(T, I)$.

If $h$ is Hénon map of degree $\lambda$ a similar argument shows $\nu\left(T_{h}, I\right)=1-\lambda^{-1}$.

\section{Green functions on $\mathbb{P}^{1} \times \mathbb{P}^{1}$}

It is possible to describe the functions $\nu, \varepsilon, g, \psi$ on a multiprojective space $\mathbb{P}^{n_{1}} \times$ $\cdots \times \mathbb{P}^{n_{k}}$. For simplicity, we only consider the case $X=\mathbb{P}^{1} \times \mathbb{P}^{1}=\mathbb{P}_{z}^{1} \times \mathbb{P}_{w}^{1}$. Let $\pi_{z}: X \rightarrow \mathbb{P}_{z}^{1}, \pi_{w}: X \rightarrow \mathbb{P}_{w}^{1}$, denote the canonical projections and set

$$
\alpha_{a, b}:=a \alpha_{z}+b \alpha_{w}, \omega_{a, b}:=a \omega_{z}+b \omega_{w}, a, b \geq 0,
$$

where $\alpha_{z}=\pi_{z}^{\star} \alpha_{1}, \alpha_{w}=\pi_{w}^{\star} \alpha_{1}, \omega_{z}=\pi_{z}^{\star} \omega_{1}, \omega_{w}=\pi_{w}^{\star} \omega_{1}$, and $\omega_{1} \in \alpha_{1}$ is the FubiniStudy form on $\mathbb{P}^{1}$. Note that $\alpha_{a, b}$ is a Kähler class if and only if $a, b>0$.

For concrete computations, it will be convenient to use coordinates on $X$. Let

$$
\pi:\left(\mathbb{C}^{2} \backslash\{0\}\right) \times\left(\mathbb{C}^{2} \backslash\{0\}\right) \rightarrow X, \pi\left(z_{0}, z_{1}, w_{0}, w_{1}\right)=\left(\left[z_{0}: z_{1}\right],\left[w_{0}: w_{1}\right]\right),
$$

and identify $\left(z_{1}, w_{1}\right) \in \mathbb{C}^{2}$ to $\pi\left(1, z_{1}, 1, w_{1}\right) \in X$. The currents $T \in \mathcal{P}\left(\alpha_{a, b}\right)$ can be described using the class $P_{a, b}$ of bihomogeneous psh functions $\widetilde{u}$ on $\mathbb{C}^{4}$ (see $[\mathrm{G}]$ ):

$$
\widetilde{u}\left(\lambda z_{0}, \lambda z_{1}, \mu w_{0}, \mu w_{1}\right)=a \log |\lambda|+b \log |\mu|+\widetilde{u}\left(z_{0}, z_{1}, w_{0}, w_{1}\right), \lambda, \mu \in \mathbb{C} .
$$

Then $\pi^{\star} T=d d^{c} \widetilde{u}$, for some $\widetilde{u} \in P_{a, b}$ which is unique up to additive constants.

For a point $p=(x, y) \in X$ we denote by

$$
V_{x}=\pi_{z}^{-1}(x)=\{z=x\}, H_{y}=\pi_{w}^{-1}(y)=\{w=y\},
$$

the vertical, and respectively horizontal, line through $p$.

\subsection{Maximal Lelong numbers.}

Proposition 3.1. For all $p=(x, y) \in X$, we have

$$
\nu\left(\alpha_{a, b}, p\right)=a+b, \varepsilon\left(\alpha_{a, b}, p\right)=\min \{a, b\} .
$$

If $T \in \mathcal{P}\left(\alpha_{a, b}\right)$ and $\nu(T, p)=a+b$ then $T=a\left[V_{x}\right]+b\left[H_{y}\right]$. Moreover, if $T$ does not charge $V_{x}$ and $H_{y}$ then $\nu(T, p) \leq \min \{a, b\}$.

Proof. Let $T \in \mathcal{P}\left(\alpha_{a, b}\right)$. We can assume that $p=(0,0)$ and let $m=\min \{a, b\}$. The current $R_{a, b} \in \mathcal{P}\left(\alpha_{a, b}\right)$ defined by $\pi^{\star} R_{a, b}=d d^{c} \widetilde{u}_{a, b}$, where $\widetilde{u}_{a, b} \in P_{a, b}$,

$\widetilde{u}_{a, b}\left(z_{0}, z_{1}, w_{0}, w_{1}\right):=m \log \sqrt{\left|z_{1} w_{0}\right|^{2}+\left|w_{1} z_{0}\right|^{2}}+(a-m) \log \left|z_{0}\right|+(b-m) \log \left|w_{0}\right|$, shows that $\varepsilon\left(\alpha_{a, b}, p\right) \geq m$. Moreover, the measure $T \wedge R_{1,1}$ is well defined and

$$
\nu(T, p)=T \wedge R_{1,1}(\{p\}) \leq \int_{X} T \wedge R_{1,1}=\int_{X} \omega_{a, b} \wedge \omega_{1,1}=a+b .
$$

Assume now that $T$ does not charge the subvarieties $V_{x}$ and $H_{y}$. By [D3], there exist $\epsilon_{j} \searrow 0$ and currents $T_{j} \in \mathcal{P}\left(\alpha_{a, b}+\epsilon_{j} \alpha_{1,1}\right)$ with analytic singularities, so that 
$0 \leq \nu(T, q)-\nu\left(T_{j}, q\right) \leq \epsilon_{j}$ for every $q \in X$. Since $T$ does not charge $V_{x}$, the measure $T_{j} \wedge\left[V_{x}\right]$ is well defined. If $v_{j}$ is a psh potential of $T_{j}$ near $p$ then

$$
\nu\left(T_{j}, p\right) \leq \nu\left(\left.v_{j}\right|_{V_{x}}, p\right)=T_{j} \wedge\left[V_{x}\right](\{p\}) \leq \int_{X} T_{j} \wedge\left[V_{x}\right]=b+\epsilon_{j} .
$$

We replace $V_{x}$ by $H_{y}$ in this argument and let $j \rightarrow+\infty$ to get $\nu(T, p) \leq m$. By (3) it follows that $\varepsilon\left(\alpha_{a, b}, p\right) \leq m$.

Assume finally that $\nu(T, p)=a+b$. By $[\mathrm{Si}]$, we can write

$$
T=a^{\prime}\left[V_{x}\right]+b^{\prime}\left[H_{y}\right]+T^{\prime}, T^{\prime} \in \mathcal{P}\left(\alpha_{a-a^{\prime}, b-b^{\prime}}\right),
$$

where $T^{\prime}$ does not charge $V_{x}$ and $H_{y}$. By what we have already shown,

$$
a+b=\nu(T, p) \leq a^{\prime}+b^{\prime}+\min \left\{a-a^{\prime}, b-b^{\prime}\right\} .
$$

This implies that $a^{\prime}=a, b^{\prime}=b$, and $T^{\prime}=0$.

Observe that the functions $\nu, \varepsilon$ are constant here, as well as in the case of $\mathbb{P}^{n}$, because $\alpha$ is invariant under a compact group of automorphisms that acts transitively on $X$.

Note that $\operatorname{Vol}\left(\alpha_{a, b}\right)^{1 / 2}=\sqrt{2 a b}>\min \{a, b\}$, hence the upper bound given in (2) is not sharp in this case. Another obvious consequence of the previous proposition is the following:

Corollary 3.2. There is no Green function with one isotropic pole on $\mathbb{P}^{1} \times \mathbb{P}^{1}$.

We can however compute the partial Green functions with isotropic singularity $\psi_{\nu, p}$ constructed in Proposition 1.6. Assume that $p=(0,0) \in \mathbb{C}^{2} \subset X$, and let $a=b=1, \nu=\varepsilon\left(\alpha_{1,1}, p\right)=1$. A psh potential of $\omega_{1,1}$ on $\mathbb{C}^{2}$ is given by

$$
\rho\left(z_{1}, w_{1}\right)=\log \sqrt{1+\left|z_{1}\right|^{2}}+\log \sqrt{1+\left|w_{1}\right|^{2}} .
$$

Proposition 3.3. We have $\psi_{1, p}\left(z_{1}, w_{1}\right)=\log \left(\left|z_{1}\right|+\left|w_{1}\right|\right)-\rho\left(z_{1}, w_{1}\right)$ if $\left|z_{1} w_{1}\right| \leq 1$, and $\psi_{1, p}\left(z_{1}, w_{1}\right)=0$ if $\left|z_{1} w_{1}\right| \geq 1$.

Proof. We have to obtain upper estimates for psh functions $v$ on $\mathbb{C}^{2}$ which verify $v \leq \rho$ and $\nu(v, 0) \geq 1$. We do this first along a complex line $z_{1}=s \zeta, w_{1}=t \zeta$. Using the same convexity argument as in the proof of Proposition 2.6, we obtain

$$
v(s \zeta, t \zeta) \leq\left\{\begin{array}{l}
\log |\zeta|+C,|\zeta| \leq R \\
\rho(s \zeta, t \zeta),|\zeta| \geq R
\end{array}\right.
$$

Here $R=|s t|^{-1 / 2}, x=\log R$ is the solution of the equation

$$
\frac{d}{d x}\left(\log \sqrt{1+|s|^{2} e^{2 x}}+\log \sqrt{1+|t|^{2} e^{2 x}}\right)=1,
$$

and $C=\log (|s|+|t|)$ verifies $\log R+C=\rho(s R, t R)$. If $s=1, t=w_{1} / z_{1}$, we get

$$
v\left(z_{1}, w_{1}\right) \leq V\left(z_{1}, w_{1}\right)=\left\{\begin{array}{l}
\log \left(\left|z_{1}\right|+\left|w_{1}\right|\right),\left|z_{1} w_{1}\right| \leq 1 \\
\rho\left(z_{1}, w_{1}\right),\left|z_{1} w_{1}\right| \geq 1
\end{array}\right.
$$

Since $\log \left(\left|z_{1}\right|+\left|w_{1}\right|\right) \leq \rho\left(z_{1}, w_{1}\right)$ on $\mathbb{C}^{2}$, with equality when $\left|z_{1} w_{1}\right|=1$, the function $V$ is psh. It follows that $\psi_{1, p}=V-\rho$. 
Note that the (unbounded) hyperconvex domain

$$
D_{1, p}=\left\{\psi_{1, p}<0\right\}=\left\{\left(z_{1}, w_{1}\right) \in \mathbb{C}^{2}:\left|z_{1} w_{1}\right|<1\right\}
$$

does not have a pluricomplex Green function: if $v<0$ is psh on $D_{1, p}$ and $v(0,0)=$ $-\infty$ then $v=-\infty$ along the lines $\left\{z_{1}=0\right\},\left\{w_{1}=0\right\}$.

3.2. Green functions with one pole. It is clear from Proposition 3.1 and Corollary 3.2 that the characterization of Green functions in $P S H\left(X, \omega_{a, b}\right)$ with one pole at $p \in X$ is more involved. Using a birational map, we will show that they correspond to a certain class of Green functions with three poles on $\mathbb{P}^{2}$. A rich class of examples of the latter can be constructed using (4) (see also Theorem 2.4). This will show that the Green functions of $X$ with pole at $p$ have many different types of singularities, even if one asks that the Lelong number at $p$ is maximal.

We may assume that $p=(0,0) \in \mathbb{C}^{2} \subset X$ and $a=1 \leq b$. Let $\omega=\omega_{F S}$ on $\mathbb{P}^{2}$ and consider the rational map $\Phi: \mathbb{P}^{2} \rightarrow X$ defined by

$$
\Phi\left(\left[t_{0}: t_{1}: t_{2}\right]\right)=\left(\left[t_{0}: t_{1}\right],\left[t_{0}: t_{2}\right]\right) .
$$

It is a birational map, with rational inverse

$$
\Phi^{-1}\left(\left[z_{0}: z_{1}\right],\left[w_{0}: w_{1}\right]\right)=\left[z_{0} w_{0}: z_{1} w_{0}: w_{1} z_{0}\right] .
$$

Note that $\Phi$ is the identity on $\mathbb{C}^{2} \equiv\left\{\left[1: t_{1}: t_{2}\right] \in \mathbb{P}^{2}\right\} \equiv\left\{\left(\left[1: z_{1}\right],\left[1: w_{1}\right]\right) \in X\right\}, \Phi$ blows up the points $A=[0: 1: 0], B=[0: 0: 1]$, to the lines $\left\{z_{0}=0\right\}$, respectively $\left\{w_{0}=0\right\}$, and $\Phi$ contracts the line $\left\{t_{0}=0\right\}$ to the point $q=(\infty, \infty)$.

We denote by $\mathcal{S}_{b}$ the set of the currents $S \in \mathcal{P}\left(\alpha_{1, b}\right)$ with locally bounded potentials on $X \backslash\{p\}$ and such that $S \wedge S=2 b \delta_{p}$. A potential of $S$ is then a $\omega_{1, b}$-psh Green function on $X$ with pole at $p$.

Let $\mathcal{R}_{b}$ be the set of currents $R \in \mathcal{P}((1+b) \omega)$ on $\mathbb{P}^{2}$ whose potentials are locally bounded on $\mathbb{P}^{2} \backslash\{p, A, B\}$, have isotropic poles at $A, B$ with Lelong numbers $\nu(R, A)=b, \nu(R, B)=1$, and such that $R \wedge R=0$ on $\mathbb{P}^{2} \backslash\{p, A, B\}$. It follows that a potential $v$ of $R$ is a $(1+b) \omega$-psh Green function on $\mathbb{P}^{2}$ with poles at $p, A, B$ :

$$
R \wedge R=\left((1+b) \omega+d d^{c} v\right)^{2}=b^{2} \delta_{A}+\delta_{B}+2 b \delta_{p} .
$$

Proposition 3.4. The mapping $\Phi^{\star}: \mathcal{S}_{b} \rightarrow \mathcal{R}_{b}$ is well defined and bijective. Its inverse is the mapping

$$
G: R \in \mathcal{R}_{b} \mapsto\left(\Phi^{-1}\right)^{\star} R-b\left[z_{0}=0\right]-\left[w_{0}=0\right] \in \mathcal{S}_{b} .
$$

Proof. Let $S \in \mathcal{S}_{b}$ and $\widetilde{u} \in P_{1, b}$ be a potential of $S$. Then

$$
\widetilde{v}\left(t_{0}, t_{1}, t_{2}\right):=\widetilde{u}\left(t_{0}, t_{1}, t_{0}, t_{2}\right), \widetilde{v}\left(\lambda t_{0}, \lambda t_{1}, \lambda t_{2}\right)=\widetilde{v}\left(t_{0}, t_{1}, t_{2}\right)+(1+b) \log |\lambda|,
$$

is a logarithmically homogeneous potential for $R=\Phi^{\star} S$, so $R \in \mathcal{P}((1+b) \omega)$. In particular, it follows that $R$ has locally bounded potentials on $\mathbb{P}^{2} \backslash\{p, A, B\}$. Near the point $A$, assuming wlog that $\left|t_{0}\right| \leq\left|t_{2}\right|$ we have

$$
\widetilde{v}\left(t_{0}, 1, t_{2}\right)=\widetilde{u}\left(t_{0}, 1, t_{0} / t_{2}, 1\right)+b \log \left|t_{2}\right|=b \log \sqrt{\left|t_{0}\right|^{2}+\left|t_{2}\right|^{2}}+O(1) .
$$

So $R$ has potentials with an isotropic pole at $A$ and $\nu(R, A)=b$. One proves in the same way that $R$ has potentials with an isotropic pole at $B$ and $\nu(R, B)=1$. We have $R \wedge R=S \wedge S=0$ on $\mathbb{C}^{2} \backslash\{0\}$. Since $R$ has locally bounded potentials near each point of $\{t=0\} \backslash\{A, B\}$ we have $R \wedge R(\{t=0\} \backslash\{A, B\})=0$, so $R \in \mathcal{R}_{b}$. 
Conversely, let $R \in \mathcal{R}_{b}$ with logarithmically homogeneous potential $\widetilde{v}$. Then

$$
\widetilde{u}\left(z_{0}, z_{1}, w_{0}, w_{1}\right):=\widetilde{v}\left(z_{0} w_{0}, z_{1} w_{0}, w_{1} z_{0}\right)-b \log \left|z_{0}\right|-\log \left|w_{0}\right| \in P_{1, b}
$$

is a bihomogeneous potential of $G(R)$. We show that $G(R)$ has locally bounded potentials in a neighborhood of any point at infinity $\zeta \neq q$. Suppose wlog $\zeta \in\left\{z_{0}=\right.$ $0\}$. Then for $\left|z_{0}\right|$ small enough we have that $\left[z_{0}: 1: z_{0} w_{1}\right]$ is near $A$, so

$$
\widetilde{u}\left(z_{0}, 1,1, w_{1}\right)=\widetilde{v}\left(z_{0}, 1, w_{1} z_{0}\right)-b \log \left|z_{0}\right|=b \log \sqrt{1+\left|w_{1}\right|^{2}}+O(1)=O(1) .
$$

Next we study the potentials of $G(R)$ in a neighborhood of $q$. We have

$$
\widetilde{u}\left(z_{0}, 1, w_{0}, 1\right)=\widetilde{v}\left(z_{0} w_{0}, w_{0}, z_{0}\right)-b \log \left|z_{0}\right|-\log \left|w_{0}\right|,
$$

where $\left|z_{0}\right|,\left|w_{0}\right|$ are small. If $\left|w_{0} / z_{0}\right|$ is small, then $\left[w_{0}: w_{0} / z_{0}: 1\right]$ is near $B$ so

$$
\widetilde{u}\left(z_{0}, 1, w_{0}, 1\right)=\widetilde{v}\left(w_{0}, w_{0} / z_{0}, 1\right)+\log \left|z_{0}\right|-\log \left|w_{0}\right|=\log \sqrt{\left|z_{0}\right|^{2}+1}+O(1) .
$$

Similarly, $\widetilde{u}\left(z_{0}, 1, w_{0}, 1\right)=O(1)$ if $\left|z_{0} / w_{0}\right|$ is small. If $\epsilon \leq\left|w_{0} / z_{0}\right| \leq M$ then

$$
\widetilde{u}\left(z_{0}, 1, w_{0}, 1\right)=\widetilde{v}\left(w_{0}, w_{0} / z_{0}, 1\right)+\log \left(\left|z_{0}\right| /\left|w_{0}\right|\right)=O(1) .
$$

It follows that $G(R)$ has locally bounded potentials in $X \backslash\{p\}$, hence $G(R) \in \mathcal{S}_{b}$.

Since $\Phi$ is the identity on $\mathbb{C}^{2}$ and the currents in $\mathcal{R}_{b}$, resp. $\mathcal{S}_{b}$, do not charge the line(s) at infinity, we conclude by the support theorem that $\Phi^{\star}$ is bijective and $G$ is its inverse.

Example 3.5. Let $1 \leq b=m / n \in \mathbb{Q}$ and $f=\left[P_{1}: P_{2}\right]: \mathbb{P}^{2} \rightarrow \mathbb{P}^{1}$, where

$$
P_{1}\left(t_{0}, t_{1}, t_{2}\right)=t_{1}^{n k} t_{2}^{m k}, P_{2}\left(t_{0}, t_{1}, t_{2}\right)=t_{1}^{n k} t_{0}^{m k}+t_{2}^{m k} t_{0}^{n k}+t_{1} t_{2} Q\left(t_{0}, t_{1}, t_{2}\right),
$$

$k \geq 1$ is an integer, and $Q$ is a homogenous polynomial of degree $(m+n) k-2$ with $\operatorname{deg}_{t_{1}} Q \leq n k-1$ and $\operatorname{deg}_{t_{2}} Q \leq m k-1$. Note that the indeterminacy set $I_{f}=\{p, A, B\}$ and the current

$$
R_{f}:=(1+b)\left(\omega+d d^{c} g_{f}\right) \in \mathcal{R}_{b},
$$

where $g_{f}$ is the Green function associated to $f$ defined in (4). Then $S_{f}=G\left(R_{f}\right)$ has bihomogeneous potential $\widetilde{u}_{f} \in P_{1, b}$ given by

$$
\widetilde{u}_{f}\left(1, z_{1}, 1, w_{1}\right)=\frac{1}{2 n k} \log \left(\left|z_{1}^{n k} w_{1}^{m k}\right|^{2}+\left|z_{1}^{n k}+w_{1}^{m k}+z_{1} w_{1} Q\left(1, z_{1}, w_{1}\right)\right|^{2}\right),
$$

where $Q\left(1, z_{1}, w_{1}\right)=\sum_{i_{1}=0}^{n k-1} \sum_{i_{2}=0}^{m k-1} c_{i_{1} i_{2}} z_{1}^{i_{1}} w_{1}^{i_{2}}$. Depending on the vanishing order of $Q(1, \cdot)$ at the origin, one sees that the Lelong number $\nu\left(S_{f}, p\right)$ can take any value of the form $\frac{j}{n k}, 2 \leq j \leq n k$. It follows that for any rational number $r \in(0,1]$ there exist $\omega_{1, b}$-psh Green functions on $X$ with one pole at $p$ and Lelong number equal to $r$ there, but with different types of singularities at $p$.

We finally give an alternate way to construct $\omega_{1,1}$-psh Green functions on $X$ with pole at $q=(\infty, \infty)$, using currents on $\mathbb{P}^{2}$ arising from psh functions in the Lelong class $\mathcal{L}^{\star}\left(\mathbb{C}^{2}\right)$. This is the class of psh functions $v$ on $\mathbb{C}^{2}$ so that

$$
\limsup _{\|s\| \rightarrow \infty} v(s) / \log \|s\|=1 .
$$

If $R$ is the trivial extension of $d d^{c} v$ to $\mathbb{P}^{2}$ then $R \in \mathcal{P}(\omega)$. 
Proposition 3.6. Let $R \in \mathcal{P}(\omega)$ be a current with locally bounded potentials in $\mathbb{P}^{2} \backslash\left\{t_{0}=0\right\}$ and near the points $A, B$. Then the current $S=\left(\Phi^{-1}\right)^{\star} R \in \mathcal{P}\left(\alpha_{1,1}\right)$, $\nu(S, q)=1$, and $S$ has locally bounded potentials on $X \backslash\{q\}$. Moreover, we have

$$
S \wedge S=2 \delta_{q} \Longleftrightarrow R \wedge R=0 \text { on } \mathbb{P}^{2} \backslash\left\{t_{0}=0\right\} .
$$

Proof. By considering (bi)homogeneous potentials as in the proof of Proposition 3.4, it follows that $S \in \mathcal{P}\left(\alpha_{1,1}\right)$ and $S$ has locally bounded potentials on $X \backslash\{q\}$. So $S \wedge S\left(\left\{z_{0}=0\right\} \cup\left\{w_{0}=0\right\} \backslash\{q\}\right)=0$, and $S \wedge S=0$ on $\mathbb{C}^{2}$ implies $S \wedge S=2 \delta_{q}$.

Let $\nu:=\nu(S, q)$. Since $\Phi$ contracts the line $\left\{t_{0}=0\right\}$ to $q$, we have that $\Phi^{\star} S=$ $\nu\left[t_{0}=0\right]+T$, where $T \in \mathcal{P}((2-\nu) \omega)$ does not charge the line $\left\{t_{0}=0\right\}$. Note that $R=T$ on $\mathbb{C}^{2}$. By the support theorem we conclude that $R=T$, so $\nu=1$.

Proposition 3.6 shows how Green functions can be constructed on $X$ by using currents $R$ on $\mathbb{P}^{2}$ possessing the right properties at any two points $A, B$ and outside the line joining them. Indeed, we pull back $R$ by an automorphism of $\mathbb{P}^{2}$ which maps the points $[0: 1: 0],[0: 0: 1]$ to $A, B$, and then apply Proposition 3.6.

Example 3.7. The Green currents $T^{+}, T^{-}$of a Hénon map $h$ on $\mathbb{C}^{2}$ yield by the preceding considerations Green functions on $X$ with pole at $q$. More generally, let $h$ be a weakly regular polynomial endomorphism of $\mathbb{C}^{2}$ with indeterminacy set $I$ (see section 2.2.4). Then its Green current $T$ has continuous local potentials on $\mathbb{P}^{2} \backslash I$ and $T \wedge T=\sum_{s \in I} m_{s} \delta_{s}$. So $T$ yields a Green function on $X$ with pole at $q$.

\section{Del Pezzo Surfaces}

We evaluate here the functions $\nu, \varepsilon, g$ when $X$ is a (smooth) Del Pezzo surface, i.e. $\operatorname{dim}_{\mathbb{C}} X=2$ and $c_{1}(X)>0$. It is well known (see e.g. [De]) that such $X$ is biholomorphic to either $\mathbb{P}^{1} \times \mathbb{P}^{1}, \mathbb{P}^{2}$, or $\mathbb{P}^{2}$ blown up at $r$ points in general position, $1 \leq r \leq 8$. Here general position means the following:

- no three points are collinear;

- no six points lie on a conic;

- when $r=8$, the points do not lie on a cubic that is singular at one of them.

The cases $X=\mathbb{P}^{2}, X=\mathbb{P}^{1} \times \mathbb{P}^{1}$, have already been considered in Sections 2 and 3. We focus here on the case when $X$ is the blow up of $\mathbb{P}^{2}$ at 8 points in general position, which we consider to be the most interesting one. The other cases could be handled similarly. Note that the Seshadri constants $\varepsilon$ are computed in [Br].

4.1. Maximal Lelong numbers. Let $\pi: X \rightarrow \mathbb{P}^{2}$ be the blow up of $\mathbb{P}^{2}$ at 8 points $p_{1}, \ldots, p_{8}$ in general position, and let $E_{j}=\pi^{-1}\left(p_{j}\right)$ denote the exceptional divisors. We let

$$
\alpha:=c_{1}(X)=K_{X}^{-1}=\pi^{\star} \mathcal{O}(3)-\sum_{j=1}^{8} E_{j}
$$

denote the (ample) anticanonical class of $X$. It is well known [De] that $2 \alpha$ is very ample. It follows from Remark 1.2 that

$$
\nu(\alpha, x) \geq 1, \varepsilon(\alpha, x) \geq 1 / 2, \forall x \in X .
$$

We can actually be much more precise. Let $\mathcal{V}$ be the pencil of cubics in $\mathbb{P}^{2}$ passing through $p_{1}, \ldots, p_{8}$. It contains at most 12 singular cubics [De]. We let $S \subset X$ denote 
the set of the corresponding singular points, $|S| \leq 12$. These points do not belong to the exceptional divisors, by the general position assumption.

Proposition 4.1. We have

$$
\nu(\alpha, x)=\left\{\begin{array}{l}
1, \text { if } x \in X \backslash S, \\
2, \text { if } x \in S
\end{array}\right.
$$

Moreover, if $x \in S$ and $T \in \mathcal{P}(\alpha)$ does not charge the strict transform of the singular cubic in $\mathcal{V}$ passing through $x$ then $\nu(T, x) \leq 1 / 2$.

Proof. For $x \in X$ there exists a unique cubic $\mathcal{C}_{x} \in \mathcal{V}$ whose strict transform $\mathcal{C}_{x}^{\prime}$ contains $x$. (If $x \in E_{j}$ this is the cubic whose strict transform intersects $E_{j}$ at $x$.) Note that $\mathcal{C}_{x}^{\prime}$ is irreducible.

Let $T \in \mathcal{P}(\alpha)$. We assume at first that $T$ does not charge $\mathcal{C}_{x}^{\prime}$ and let $\omega$ be a fixed Kähler form on $X$. By [D3] there exist $\epsilon_{j} \searrow 0$ and currents $T_{j} \in \mathcal{P}\left(\alpha+\epsilon_{j} \omega\right)$ with analytic singularities, such that $T_{j} \rightarrow T$ and $0 \leq \nu(T, z)-\nu\left(T_{j}, z\right) \leq \epsilon_{j}$ for all $z \in X$. Since $T$ does not charge $\mathcal{C}_{x}^{\prime}$, the measure $T_{j} \wedge\left[\mathcal{C}_{x}^{\prime}\right]$ is well defined. As $\operatorname{Vol}(\alpha)=1$ it follows that

$$
1+O\left(\epsilon_{j}\right)=\int_{X} T_{j} \wedge\left[\mathcal{C}_{x}^{\prime}\right] \geq T_{j} \wedge\left[\mathcal{C}_{x}^{\prime}\right](\{x\}) \geq \nu\left(T_{j}, x\right) m\left(\mathcal{C}_{x}^{\prime}, x\right),
$$

where $m\left(\mathcal{C}_{x}^{\prime}, x\right)$ denotes the multiplicity of $\mathcal{C}_{x}^{\prime}$ at $x$. The last inequality can be seen by using a local normalization at $x$ for each irreducible component of $\mathcal{C}_{x}^{\prime}$ and since local psh potentials of $T_{j}$ are subharmonic along $\mathcal{C}_{x}^{\prime}$.

Letting $j \rightarrow+\infty$, we have shown that $\nu(T, x) \leq 1 / m\left(\mathcal{C}_{x}^{\prime}, x\right) \leq 1$, if $T \in \mathcal{P}(\alpha)$ does not charge $\mathcal{C}_{x}^{\prime}$. In particular, if $x \in S$ then $\nu(T, x) \leq 1 / 2$ since $m\left(\mathcal{C}_{x}^{\prime}, x\right)=2$.

In the general case, we can write by $[\mathrm{Si}]$

$$
T=a\left[\mathcal{C}_{x}^{\prime}\right]+(1-a) R, 0 \leq a \leq 1,
$$

where $R \in \mathcal{P}(\alpha)$ does not charge $\mathcal{C}_{x}^{\prime}$. Then

$$
\nu(T, x)=\operatorname{am}\left(\mathcal{C}_{x}^{\prime}, x\right)+(1-a) \nu(R, x) \leq a\left(m\left(\mathcal{C}_{x}^{\prime}, x\right)-1\right)+1 \leq m\left(\mathcal{C}_{x}^{\prime}, x\right),
$$

which concludes the proof.

4.2. Uniform integrability exponent. We fix $\omega \in \alpha=c_{1}(X)$ a Kähler form and we denote by $P S H_{0}(X, \omega)$ the set of $\omega$-psh functions $\varphi$ normalized by $\max _{X} \varphi=0$. This is a compact subset of $L^{1}(X)$. Set

$$
\sigma(X)=\sup \left\{c \geq 0: e^{-2 c \varphi} \in L^{1}(X), \forall \varphi \in P S H_{0}(X, \omega)\right\} .
$$

This number clearly depends only on $\alpha=c_{1}(X)$, rather than on the particular choice of $\omega$. By the compactness of $P S H_{0}(X, \omega)$ and the semicontinuity of the "complex singularity exponent" $[\mathrm{DK}], \sigma(X)$ coincides with the exponent introduced by Tian in $[\mathrm{T}]$ (the so-called " $\alpha$-invariant of Tian").

We assume here again that $X$ is the blow up of $\mathbb{P}^{2}$ at 8 points in general position. Since $\nu(\alpha, x) \leq 2$ for all $x \in X$, it follows from Skoda's integrability theorem [Sk] that $\sigma(X) \geq 1 / 2$. One can however obtain sharp estimates, thanks to the full characterization given in Proposition 4.1:

Proposition 4.2. If there is a singular cubic in $\mathcal{V}$ with a cusp then $\sigma(X)=5 / 6$. Otherwise, $\sigma(X)=1$. 
Recall that there is no cuspidal cubic in $\mathcal{V}$ when the points $p_{1}, \ldots, p_{8}$ are in very general position [De].

Proof of Proposition 4.2. Let $s=|S| \leq 12$ and $\mathcal{C}_{j}^{\prime}, 1 \leq j \leq s$, denote the strict transforms of the singular cubics in $\mathcal{V}$. We write $\left[\mathcal{C}_{j}^{\prime}\right]=\omega+d d^{c} \varphi_{j}$, where $\varphi_{j} \in$ $P S H_{0}(X, \omega)$.

Fix now $\varphi \in P S H_{0}(X, \omega)$ and let $T=\omega+d d^{c} \varphi \in \mathcal{P}(\alpha)$. By [Si],

$$
T=a_{0} T_{0}+\sum_{j=1}^{s} a_{j}\left[\mathcal{C}_{j}^{\prime}\right], \text { where } a_{j} \geq 0, \sum_{j=0}^{s} a_{j}=1,
$$

and $T_{0}=\omega+d d^{c} \varphi_{0} \in \mathcal{P}(\alpha)$ does not charge any curve $\mathcal{C}_{j}^{\prime}$. Hölder's inequality shows that $e^{-2 c \varphi} \in L^{1}(X)$ if $e^{-2 c \varphi_{j}} \in L^{1}(X)$ for all $j=0, \ldots, s$.

For $j \geq 1$, a direct computation in local coordinates shows that $e^{-2 c \varphi_{j}} \in L^{1}(X)$ for every $c<1$ if $\mathcal{C}_{j}^{\prime}$ is non-singular or has a simple node, while $e^{-2 c \varphi_{j}} \in L^{1}(X)$ for every $c<5 / 6$ if $\mathcal{C}_{j}^{\prime}$ has a cusp. In the latter case, $e^{-2 c \varphi_{j}} \notin L^{1}(X)$ if $c=5 / 6$.

Since $T_{0}$ does not charge any curve $\mathcal{C}_{j}^{\prime}$, it follows from Proposition 4.1 that $\nu\left(T_{0}, x\right) \leq 1$ for all $x \in X$. By [Sk] we see that $e^{-2 c \varphi_{0}} \in L^{1}(X)$ for every $c<1$. This completes the proof of the proposition.

Note that $\sigma(X)$ is also called the (global) "log-canonical threshold" of $X$. It has been the subject of intensive studies in the last decade. The above result has been recently obtained by Cheltsov $[\mathrm{Ch}]$ by more algebraic methods.

The importance of this notion is seen in its connection with the existence of Kähler-Einstein metrics: it was shown by Tian $[\mathrm{T}]$ that a Fano surface admits a Kähler-Einstein metric if $\sigma(X)>2 / 3$. The exponent $\sigma(X)$ was previously estimated by Tian and Yau in [TY].

4.3. Green functions. In this section $X$ denotes again the blow up of $\mathbb{P}^{2}$ at 8 points in general position.

4.3.1. Special points. For $x \in S$, let $\mathcal{C}_{x}$ be the cubic in $\mathcal{V}$ which is singular at $x$, and let $\mathcal{C}_{x}^{\prime}$ be its strict transform.

Counting dimension we see that there exists an irreducible sextic $Z \subset \mathbb{P}^{2}$ passing through $x$ and with multiplicity 2 at each point $p_{j}$. By Bezout we see that $Z$ and $\mathcal{C}_{x}$ intersect only at $x$ and at the points $p_{j}$ and the intersection numbers $\left(Z \cdot \mathcal{C}_{x}\right)_{p_{j}}=$ $\left(Z \cdot \mathcal{C}_{x}\right)_{x}=2$. This implies that the strict transform $Z^{\prime} \subset X$ of $Z$ intersects $\mathcal{C}_{x}^{\prime}$ only at $x$ with $\left(Z^{\prime} \cdot \mathcal{C}_{x}^{\prime}\right)_{x}=2$.

We write $(1 / 2)\left[Z^{\prime}\right]=\omega+d d^{c} u,\left[\mathcal{C}_{x}^{\prime}\right]=\omega+d d^{c} v$, and set

$$
g_{x}:=(1 / 2) \log \left(e^{2 u}+e^{2 v}\right) \in P S H(X, \omega) \cap C^{\infty}(X \backslash\{x\}) .
$$

Proposition 4.3. If $x \in S$ we have $\left(\omega+d d^{c} g_{x}\right)^{2}=\delta_{x}$, and the function $g_{x}$ is a $\omega$-psh Green function with Lelong number $\nu\left(g_{x}, x\right)=1 / 2$.

Proof. Since $Z^{\prime}$ is smooth at $x$ we have $\nu\left(g_{x}, x\right)=1 / 2$. Moreover, $\left(Z^{\prime} \cdot \mathcal{C}_{x}^{\prime}\right)_{x}=2$ implies that $\left(\omega+d d^{c} g_{x}\right)^{2}(\{x\})=1$. We conclude by mass considerations.

Observe that the singularity of $g_{x}$ at $x$ is not isotropic, since an isotropic pole with Lelong number $1 / 2$ would produce a Dirac mass at $x$ with coefficient $1 / 4$. However, the existence of a Green function which is locally bounded away from $x$ has interesting consequences: 
Corollary 4.4. If $x \in S$ then $\varepsilon(\alpha, x)=1 / 2$. Moreover, the supremum is attained in the formula (3) of $\varepsilon(\alpha, x)$, i.e.

$$
\exists \varphi \in P S H(X, \omega) \cap L_{l o c}^{\infty}(X \backslash\{x\}),\|\varphi-(1 / 2) \log \operatorname{dist}(\cdot, x)\|_{L^{\infty}(X)}<+\infty .
$$

Proof. It follows from (6) and Proposition 4.1 that $\varepsilon(\alpha, x)=1 / 2$. Let $g_{x}$ be the function constructed in Proposition 4.3. Fix $\chi \in C^{\infty}(X)$ a test function with $\chi \equiv 1$ on $\bar{U}$, where $U$ is a small open neighborhood of $x$. We define

$$
\varphi:=\max \left\{g_{x},(1 / 2) \chi \log \operatorname{dist}(\cdot, x)-C\right\},
$$

where $C$ is large so that $\varphi=g_{x}$ on $X \backslash U$. Since $\chi \log \operatorname{dist}(\cdot, x)$ is psh on $U$ we see that $\varphi \in \operatorname{PSH}(X, \omega)$. Now $\nu\left(g_{x}, x\right)=1 / 2$, therefore $\varphi-(1 / 2) \log \operatorname{dist}(\cdot, x)$ is bounded on $X$.

4.3.2. Generic points. Assume now that $x \in X \backslash S$. The bound (6) is not sharp: by [Br] we have $\varepsilon(\alpha, x)=1$.

It is easy to see that the supremum in formula (3) is attained if $x$ is the ninth base point of the pencil of cubics $\mathcal{V}$. In this case we write $\left[\mathcal{C}_{1}^{\prime}\right]=\omega+d d^{c} u,\left[\mathcal{C}_{2}^{\prime}\right]=\omega+d d^{c} v$, where $\mathcal{C}_{j}^{\prime}$ are the strict transforms of two cubics generating $\mathcal{V}$, and we set

$$
g_{x}:=(1 / 2) \log \left(e^{2 u}+e^{2 v}\right) \in P S H(X, \omega) \cap C^{\infty}(X \backslash\{x\}) .
$$

We have that $\left(\omega+d d^{c} g_{x}\right)^{2}=\delta_{x}$ and $g_{x}$ is a $\omega$-psh Green function with an isotropic pole at $x$ with $\nu\left(g_{x}, x\right)=1$.

However, it is unclear whether this holds at arbitrary points $x \in X \backslash S$. If this was the case, it would imply that $K_{Y}^{-1}$ admits a positive metric with bounded potentials, where $Y \rightarrow \mathbb{P}^{2}$ is the blow up of $\mathbb{P}^{2}$ at 9 points in general position, which is a famous open problem (see [DPS]). Observe that the existence of such a metric is equivalent to constructing a $\omega_{F S}$-psh Green function with isotropic poles of Lelong number $1 / 3$ at 9 points in general position in $\mathbb{P}^{2}$.

More generally, finding a $\omega_{F S}$-psh Green function with isotropic poles of Lelong number $1 / \sqrt{s}$ at $s$ points in general position in $\mathbb{P}^{2}$ is equivalent to the celebrated (strong version of) Nagata's conjecture (see [La, Remark 5.1.14]).

\section{REFERENCES}

[BT1] E. Bedford and B. A. Taylor, The Dirichlet problem for a complex Monge-Ampère equation, Invent. Math. 37 (1976), 1-44.

[BT2] E. Bedford and B. A. Taylor, A new capacity for plurisubharmonic functions, Acta Math. 149 (1982), 1-40.

[BGZ] S. Benelkourchi, V. Guedj and A. Zeriahi, A priori estimates for weak solutions of complex Monge-Ampère equations, Ann. Scuola Norm. Sup. Pisa Cl. Sci. (5) 7 (2008), $1-16$.

[BP] Z. Błocki and P. Pflug, Hyperconvexity and Bergman completeness, Nagoya Math. J. 151 (1998), 221-225.

[Br] A. Broustet, Constantes de Seshadri du diviseur anticanonique des surfaces de del Pezzo, Enseign. Math. (2) 52 (2006), 231-238.

[CP] H. I. Celik and E. A. Poletsky, Fundamental solutions of the complex Monge-Ampère equation, Ann. Polon. Math. 67 (1997), 103-110.

[Ch] I. Cheltsov, Log canonical thresholds of Del Pezzo surfaces, Geom. Funct. Anal. 18 (2008), 1118-1144.

[Co1] D. Coman, Certain classes of pluricomplex Green functions on $\mathbb{C}^{n}$, Math. Z. 235 (2000), 111-122. 
[Co2] D. Coman, Entire pluricomplex Green functions and Lelong numbers of projective currents, Proc. Amer. Math. Soc. 134 (2006), 1927-1935.

[CGZ] D. Coman, V. Guedj, and A. Zeriahi, Domains of definition of Monge-Ampère operators on compact Kähler manifolds, Math. Z. 259 (2008), 393-418.

[CN] D. Coman and S. Nivoche, Plurisubharmonic functions with singularities and affine invariants for finite sets in $\mathbf{C}^{n}$, Math. Ann. 322 (2002), 317-332.

[D1] J. P. Demailly, Mesures de Monge-Ampère et mesures plurisousharmoniques, Math. Z. 194 (1987), 519-564.

[D2] J. P. Demailly, Singular Hermitian metrics on positive line bundles, in Complex algebraic varieties (Bayreuth, 1990), Lecture Notes in Math. 1507, Springer, Berlin, 1992, 87-104.

[D3] J. P. Demailly, Regularization of closed positive currents and intersection theory, J. Algebraic Geom. 1 (1992), 361-409.

[D4] J. P. Demailly, Monge-Ampère operators, Lelong numbers and intersection theory, in Complex analysis and geometry, Plenum, New York, 1993, 115-193.

[D5] J. P. Demailly, A numerical criterion for very ample line bundles, J. Differential Geom. 37 (1993), 323-374.

[DK] J. P. Demailly and J. Kollár, Semi-continuity of complex singularity exponents and Kähler-Einstein metrics on Fano orbifolds, Ann. Sci. École Norm. Sup. (4) 34 (2001), $525-556$.

[DP] J. P. Demailly and M. Paun, Numerical characterization of the Kähler cone of a compact Kähler manifold, Ann. of Math. (2) 159 (2004), 1247-1274.

[DPS] J. P. Demailly, T. Peternell and M. Schneider, Compact Kähler manifolds with Hermitian semipositive anticanonical bundle, Compositio Math. 101 (1996), 217-224.

[De] M. Demazure, Surfaces de Del Pezzo. I-V, Sémin. sur les singularités des surfaces, Cent. Math. Ec. Polytech., Palaiseau 1976-77, Lecture Notes in Math. 777 (1980), 21-69.

[DH] K. Diederich and G. Herbort, Quantitative estimates for the Green function and an application to the Bergman metric, Ann. Inst. Fourier (Grenoble) 50 (2000), 1205-1228.

[EGZ] P. Eyssidieux, V. Guedj and A. Zeriahi, Singular Kähler-Einstein metrics, J. Amer. Math. Soc., to appear.

[FH] W. Fulton and J. Hansen, A connectedness theorem for projective varieties, with applications to intersections and singularities of mappings, Ann. of Math. (2) 110 (1979), 159-166.

[G] V. Guedj, Dynamics of polynomial mappings of $\mathbb{C}^{2}$, Amer. J. Math. 124 (2002), 75-106.

[GS] V. Guedj and N. Sibony, Dynamics of polynomial automorphisms of $\mathbb{C}^{k}$, Ark. Mat. 40 (2002), 207-243.

[GZ1] V. Guedj and A. Zeriahi, Intrinsic capacities on compact Kähler manifolds, J. Geom. Anal. 15 (2005), 607-639.

[GZ2] V. Guedj and A. Zeriahi, The weighted Monge-Ampère energy of quasiplurisubharmonic functions, J. Funct. Anal. 250 (2007), 442-482.

[He] G. Herbort, The Bergman metric on hyperconvex domains, Math. Z. 232 (1999), 183196.

[Ho] L. Hörmander, Notions of Convexity, Birkhäuser, 1994.

[Kl] M. Klimek, Extremal plurisubharmonic functions and invariant pseudodistances, Bull. Soc. Math. France 113 (1985), 231-240.

[Ko1] S. Kołodziej, The complex Monge-Ampère equation, Acta Math. 180 (1998), 69-117.

[Ko2] S. Kołodziej, The set of measures given by bounded solutions of the complex MongeAmpère equation on compact Kähler manifolds, J. London Math. Soc. (2) 72 (2005), $225-238$.

[La] R. Lazarsfeld, Positivity in algebraic geometry. I. Classical setting: line bundles and linear series, Results in Mathematics and Related Areas. 3rd Series. A Series of Modern Surveys in Mathematics, 48, Springer-Verlag, Berlin, 2004.

[Le] P. Lelong, Fonction de Green pluricomplexe et lemmes de Schwarz dans les espaces de Banach, J. Math. Pures Appl. (9) 68 (1989), 319-347.

[Lm] L. Lempert, Solving the degenerate complex Monge-Ampère equation with one concentrated singularity, Math. Ann. 263 (1983), 515-532. 
[R] A. Rashkovskii, Relative types and extremal problems for plurisubharmonic functions, Int. Math. Res. Not. 2006, Art. ID 76283, 26 pp.

[S] N. Sibony, Dynamique des applications rationnelles de $\mathbb{P}^{k}$, Panoramas et Synthèses 8 (1999), 97-185.

[Si] Y. T. Siu, Analyticity of sets associated to Lelong numbers and the extension of closed positive currents, Invent. Math. 27 (1974), 53-156.

[Sk] H. Skoda, Sous-ensembles analytiques d'ordre fini ou infini dans $\mathbb{C}^{n}$, Bull. Soc. Math. France 100 (1972), 353-408.

[T] G. Tian, On Kähler-Einstein metrics on certain Kähler manifolds with $C_{1}(M)>0$, Invent. Math. 89 (1987), 225-246.

[TY] G.Tian and S.T.Yau: Kähler-Einstein metrics on complex surfaces with $C_{1}>0$. Comm. Math. Phys. 112 (1987), no. 1, 175-203.

[Y] S. T. Yau, On the Ricci curvature of a compact Kähler manifold and the complex MongeAmpère equation I, Comm. Pure Appl. Math. 31 (1978), 339-411.

D. Coman: dcoman@syr.edu, Department of Mathematics, Syracuse University, SyRaCUSE, NY 13244-1150, USA

V. Guedj: Guedj@Cmi.univ-mrs.fr, Université Aix-Marseille 1, LATP, 13453 MarSEILle CEDEX 13, FRANCE 